\title{
Merging history of three bimodal clusters ${ }^{\star} \star \star \star$
}

\author{
S. Maurogordato ${ }^{1}$, J. L. Sauvageot ${ }^{2}$, H. Bourdin ${ }^{3}$, A. Cappi ${ }^{1,4}$, C. Benoist ${ }^{1}$, C. Ferrari ${ }^{1}$, G. Mars ${ }^{1}$, and K. Houairi ${ }^{5,6}$ \\ 1 Université de Nice Sophia-Antipolis, CNRS, Observatoire de la Côte d’Azur, UMR 6202 Cassiopée, BP 4229, \\ 06304 Nice Cedex 4, France \\ e-mail: Sophie.Maurogordato@oca.eu \\ 2 CEA, DSM, Irfu, Service d'Astrophysique, C.E. Saclay, 91191 Gif-sur-Yvette Cedex, France \\ 3 Dipartimento di Fisica, Università degli Studi di Roma "Tor Vergata", via della Ricerca Scientifica, 1, 00133 Roma, Italy \\ ${ }^{4}$ INAF - Osservatorio Astronomico di Bologna, via Ranzani 1, 40127 Bologna, Italy \\ 5 ONERA, Optics Department, BP 72, 92322 Chatillon Cedex, France \\ ${ }^{6}$ CNES, 18 avenue Édouard Belin, 31401 Toulouse Cedex 09, France
}

Received 12 March 2010 / Accepted 20 August 2010

\section{ABSTRACT}

\begin{abstract}
We present a combined X-ray and optical analysis of three bimodal galaxy clusters selected as merging candidates at $z \sim 0.1$. These targets are part of MUSIC (MUlti-Wavelength Sample of Interacting Clusters), which is a general project designed to study the physics of merging clusters by means of multi-wavelength observations. Observations include spectro-imaging with XMM-Newton EPIC camera, multi-object spectroscopy (260 new redshifts), and wide-field imaging at the ESO $3.6 \mathrm{~m}$ and $2.2 \mathrm{~m}$ telescopes. We build a global picture of these clusters using X-ray luminosity and temperature maps together with galaxy density and velocity distributions. Idealized numerical simulations were used to constrain the merging scenario for each system. We show that A2933 is very likely an equal-mass advanced pre-merger $\sim 200 \mathrm{Myr}$ before the core collapse, while A2440 and A2384 are post-merger systems ( 450 Myr and $\sim 1.5$ Gyr after core collapse, respectively). In the case of A2384, we detect a spectacular filament of galaxies and gas spreading over more than $1 h^{-1} \mathrm{Mpc}$, which we infer to have been stripped during the previous collision. The analysis of the MUSIC sample allows us to outline some general properties of merging clusters: a strong luminosity segregation of galaxies in recent post-mergers; the existence of preferential axes - corresponding to the merging directions - along which the BCGs and structures on various scales are aligned; the concomitance, in most major merger cases, of secondary merging or accretion events, with groups infalling onto the main cluster, and in some cases the evidence of previous merging episodes in one of the main components. These results are in good agreement with the hierarchical scenario of structure formation, in which clusters are expected to form by successive merging events, and matter is accreted along large-scale filaments.
\end{abstract}

Key words. X-rays: galaxies: clusters - galaxies: clusters: intracluster medium - galaxies: clusters: individual: Abell 2384 galaxies: clusters: individual: Abell 2440 - galaxies: clusters: individual: Abell 2933

\section{Introduction}

In standard Cold Dark Matter cosmological models, including the concordance $\Lambda \mathrm{CDM}$, the general growth of structures starts from the primordial density fluctuations generated by inflation, and is driven by gravity in a hierarchical way, i.e. smaller structures form first, then merge into progressively more massive systems; however, if the acceleration of the expansion is due to a cosmological constant, the process of structure formation will completely stop in the future (Krauss \& Starkman 2000; Nagamine \& Loeb 2003; Busha et al. 2005).

Galaxy clusters are the largest gravitationally bound objects of the hierarchy; they accrete smaller groups coming from the filaments of the cosmic web and occasionally merge with other clusters of comparable mass, releasing an exceptionally high amount of energy. Merging clusters are therefore ideal laboratories to study the process of structure formation and how its affects galaxy evolution. In this scenario, one expects to find

* Based on data obtained with the European Southern Observatory, Chile (programs 072.A-0595, 075.A-0264, and 079.A-0425).

$\star \star$ Tables 5-7 are only available in electronic form at the CDS via anonymous ftp to cdsarc.u-strasbg.fr $(130.79 .128 .5)$ or via http://cdsarc.u-strasbg.fr/viz-bin/qcat?J/A+A/525/A79 a large fraction of clusters in their formation process at high redshift (which is corroborated by the large fraction of irregular morphologies observed). However, observations of clusters at high redshift are difficult and time consuming, so an alternative choice is to search for rarer but more easily observable merging candidates at low redshift. In this way, we can probe in detail the merging signatures and try to shed some light on the process of cluster formation and how it affects galaxy evolution.

A crucial progress in the study of merging clusters was provided by the spectral and imaging capabilities of the last generation of X-ray satellites. Before then, X-ray spectroscopic information had not been available or of very poor quality, and the main information about mergers was based on morphology. With the spatially resolved spectroscopy and high resolution imaging offered by Chandra and XMM, the situation has radically changed, and sophisticated algorithms have been developed to achieve good and reliable temperature maps (see for instance Bourdin et al. 2004), since the temperature is the more accurate tracer of the energy transfer from the collision to the X-ray gas itself. Strong signatures of the merging events have been detected in these maps (Vikhlinin et al. 2000; Markevitch et al. 2000 and 2002), and cold fronts and bow shocks are now well 
established as common merger features. Thanks to this observational progress, major mergers of galaxy clusters now appear far more complex than previously foreseen.

The full understanding of the complex processes at work in merging requires dedicated numerical simulations. Much progress has been made in this field, starting from the pioneering works of Schindler \& Böhringer (1993) and Roettiger et al. (1997). Ricker \& Sarazin (2001, hereafter RS01) described the violent relaxation of gas in a dark matter potential well for a variety of idealized merging systems, paying special attention to the impact parameter and the mass ratio between units. Poole et al. (2006) analyzed merging of idealized relaxed clusters with sophisticated SPH simulations including cooling and star formation, and detected the major transient signatures existing in observed temperature maps.

Combining optical with X-ray data has been shown to be extremely effective in unveiling the complex history of merging clusters (to mention a few, Arnaud et al. 2000; Maurogordato et al. 2000; Donnelly et al. 2001; Barrena et al. 2002, 2007; Boschin et al. 2004; Owers et al. 2009). These studies have revealed various peculiar properties of the galaxy distribution in the individual merging clusters, such as strong signatures in the density and velocity distribution, and strong alignments effects. However, a larger sample with both X-ray and optical observations is clearly needed to test the generality of these properties, and their dependence on the merging stage.

Motivated by these reasons, we started an observational program, MUSIC (MUltiwavelength Sample of Interacting Clusters) to define and analyze a sample of merging clusters at different stages of the merging process. The first targets (A3921, A1750, A2065, and A2255) were selected from the merging clusters observed during the XMM Guaranteed Time by one of us (Sauvageot), and we established an optical follow-up program at ESO.

To cover systems representing various stages of merging, we extended the initial sample by including targets from Kolokotronis et al. (2001) which compared the density distributions of the gas (from ROSAT/HRI maps) and of the galaxies (from APM maps). We selected the systems with a clear bimodal morphology in both the X-ray and the optical maps as probable pre-merger candidates, and those with a distorted morphology and a pronounced segregation between gas (collisional) and galaxies (non collisional), i.e. with features that are expected of mergers at a more advanced stage. We selected clusters around $z \sim 0.1$, because at this redshift evolutionary effects on galaxies are negligible and the field of view of X-ray and optical instruments offers a good coverage of the system (30' with XMM and wide field imagers such as WFI at the ESO $2.2 \mathrm{~m}$, corresponding to $2.3 h^{-1} \mathrm{Mpc}$ at $z=0.1$ ). With a $4 \mathrm{~m}$ class telescope, spectroscopy is also easily performed as faint as $R<19$, a limit corresponding to $\sim L_{*}+3.5$ at this redshift (with a best-fit parameter $M_{R}^{*}=-22.97$ estimated by Popesso et al. (2005), fitting a Schechter function to the cluster galaxy luminosity function of the RASS-SDSS cluster survey). The aims of the program are to characterize the merging scenario (epoch of collision, geometry, and mass ratios) and to test the impact of the merging process on the properties of galaxies, in particular the star formation. For example, the combined X-ray and optical analysis allowed us to determine the merging scenario of the first two clusters (a premerger for A1750, Belsole et al. 2004; and a recent post-merger for A3921, Belsole et al. 2005; Ferrari et al. 2005).

Here we present the analysis of three additional merging candidates: A2933, A2440, and A2384, based on XMM X-ray spectro-imaging obtained in 2005 and 2006 (A2933 and A2440), or retrieved from the XSA database (A2384) and optical observations carried out in 2003, 2005, and 2007 at ESO, including wide-field multi-band imaging with the Wide Field Imager on the $2.2 \mathrm{~m}$ telescope, and multi-object spectroscopy with EFOSC2 at the $3.6 \mathrm{~m}$ telescope.

The clusters A2933, A2440 and A2384 share common features: an irregular morphology (they have been classified as Bautz-Morgan type III, II, and II/III, respectively), low Abell richness classes $(1,0$, and 1$)$, and redshifts slightly less than $0.1(0.0925,0.0906$, and 0.0943 according to Struble \& Rood 1999). In the optical, A2933 was observed as a target of the ESO Nearby Abell Cluster Survey (Katgert et al. 1996) and the southern Abell redshift survey (Muriel et al. 2002; Way et al. 2005; Coenda et al. 2006). A2440 was identified as a pre-merging cluster from the dynamical and X-ray/optical analysis of Beers et al. (1991) and Mohr et al. (1996). A2384 is also a classical example of a bimodal cluster (Ulmer \& Cruddace 1982; West et al. 1995). A weak-lensing analysis of A2384 was performed by Cypriano et al. (2004). Both A2440 and A2384 belong to the flux-limited sample of bright clusters of galaxies from the southern part of the ROSAT All-Sky Survey (de Grandi et al. 1999).

In Sects. 2 and 3, we present the data, the reduction procedure, and the methodology used in X-ray and optical respectively. In Sects. 4-6, we analyze the data for A2933, A2440, and A2384 respectively and propose a merging scenario for each cluster. In Sect. 7 we present our numerical simulations of merging clusters that are used in Sect. 8 to refine the scenarios. In the following, we adopt the cosmological parameters of a $\Lambda \mathrm{CDM}$ model with $\Omega_{\mathrm{M}}=0.3, \Omega_{\Lambda}=0.7$, and $H_{0}=70 \mathrm{~km} \mathrm{~s}^{-1} \mathrm{Mpc}^{-1}$. With these parameters, at $z=0.1$ one degree corresponds to a physical length of $6.6 \mathrm{Mpc}$.

\section{X-ray observations: scientific products}

The three systems presented in this paper were observed by XMM-Newton EPIC cameras for about $30 \mathrm{ks}$ in full frame mode with the medium filter. The observation were screened for proton flares on a high and low energy light curve basis. A summary of the effective exposure time remaining after this screening process is provided in Table 1.

The brightness of the X-ray emitting intra-cluster medium (ICM) was mapped from a multi-scale algorithm using Haar wavelets, especially suited to denoise images dominated by shot noise. First proposed by Jammal \& Bijaoui (2004), this algorithm was adapted to analyze X-ray astronomical images and correct signal distorsions related to the spatially variable detector response, and mirror effective area (Bourdin et al. 2008). We estimated the ICM brightness from the spatial distribution of low energy X-ray photons (with energy lower than $2.5 \mathrm{keV}$ ), since their spectral distribution is expected to have a weak dependence on temperature.

We used the X-ray data set in order to map the ICM temperature. To do so, we used the wavelet spectral-imaging algorithm proposed in Bourdin et al. (2004) and Bourdin et al. (2008). X-ray spectral-imaging can be performed only on data of sufficiently high count statistics, and it is necessary to gather photons within large detector regions, the shape of which needs to be optimised to detect thermal structure. To overcome this major difficulty, we developed a strategy based on wavelet transforms, where we first estimated the ICM temperature with its associated confidence range at various angular scales and locations in the field of view. The detection of the temperature features and the reconstruction of an ICM temperature map was finally obtained by applying a threshold to the wavelet coefficients. The wavelet 
S. Maurogordato et al.: Merging history of three bimodal clusters

Table 1. XMM-Newton-EPIC observations used in our analysis, with effective exposure time corresponding to each instrument.

\begin{tabular}{cccccc}
\hline \hline Cluster name & $\begin{array}{c}\text { XMM-Newton } \\
\text { obs. IDs }\end{array}$ & Center coordinates & $\begin{array}{c}\text { MOS1 effective } \\
\text { exposure time (ks) }\end{array}$ & $\begin{array}{c}\text { MOS2 effective } \\
\text { exposure time (ks) }\end{array}$ & $\begin{array}{c}\text { PN effective } \\
\text { exposure time (ks) }\end{array}$ \\
\hline A2933 & 0305060101 & $01^{\mathrm{h}} 40^{\mathrm{m}} 41.2^{\mathrm{s}}-54^{\circ} 33^{\prime} 26.0^{\prime \prime}$ & $21.5(69.3 \%)$ & $27.7(89.0 \%)$ & $16.3(68.5 \%)$ \\
A2440 & 0401920101 & $22^{\mathrm{h}} 23^{\mathrm{m}} 52.6^{\mathrm{s}}-01^{\circ} 36^{\prime} 57.0^{\prime \prime}$ & $25.3(55.4 \%)$ & $24.9(54.3 \%)$ & $17.8(50.3 \%)$ \\
A2384 & 0101902701 & $21^{\mathrm{h}} 52^{\mathrm{m}} 14.2^{\mathrm{s}}-19^{\circ} 42^{\prime} 19.8^{\prime \prime}$ & $17.2(65.4 \%)$ & $16.8(63.6 \%)$ & $10.2(51.6 \%)$ \\
\hline
\end{tabular}

Notes. In brackets: fraction of the useful exposure time after solar-flare "cleaning".

analysis was performed following a shift-invariant algorithm using B-spline wavelet coefficients, and applying a threshold of $1 \sigma$ confidence level above the noise fluctuation. Point sources have to be masked before building the map, to avoid pollution from the ICM signal at different spatial frequencies.

\section{Optical observations: data description and reduction methods}

All three clusters were allocated time at ESO for wide-field imaging with the WFI instrument at the $2.2 \mathrm{~m}$ telescope (total of $9.75 \mathrm{~h}$ ), and extensive multi-object spectroscopy with EFOSC2 at the $3.6 \mathrm{~m}$ telescope (total of 6 nights), in 2003, 2005, and 2007, respectively (programs 072.A-0595, 075.A-0264, and 079.A-0425). As wide-field imaging of A2933 in service mode was not performed, we used the APM galaxy survey (Maddox et al. 1990) and the DSS images in the analysis of this cluster. We retrieved the relevant galaxy catalog in a region of $30^{\prime} \times 30^{\prime}$ covering A2933 from the Southern sky catalogue based on the UKST SES $R$ survey. For A2384 and A2440, we obtained imaging of the central $30^{\prime} \times 30^{\prime}$ field in the $R$ (filter ESO/844) and $B$ (filter ESO/878) passbands. For each filter, 8 dithered images were obtained leading to a total exposure time of $40 \mathrm{~min}$. These sets of images were reduced and combined using the ESO/MVM package Alambic (Vandamme et al. 2002), and the catalogs were extracted with SExtractor (Bertin \& Arnouts 1996). The typical seeing is 1 arcsec. The magnitudes used in the present paper are the total magnitudes " $M A G_{\text {AUTO" }}$ " provided by SExtractor. Stars and galaxies were classified (up to a magnitude of 21 in both bands) by extracting the stellar sequences within the magnitudehalf light radius diagrams. Fainter objects were all considered as galaxies. Finally, catalogs with $B-R$ color were built by associating objects with positions in the $B$ and $R$ catalogs differing by less than 1.5 arcsec.

For A2440 and A2384, the final catalog of galaxies including both $B$ and $R$ passbands and covering a field of $30^{\prime} \times 30^{\prime}$ was used to analyze the color properties and the spatial distribution of the cluster galaxies. The color-magnitude diagram was used to identify the red sequence defined by the cluster elliptical population (Lopez-Cruz et al. 2004). We selected galaxies with colors within $3 \sigma$ of this relation as high probability members of the cluster and from this selection we derived the projected density maps for different magnitude cuts in the $R$-band (corresponding typically to $L^{*}+1, L^{*}+2, L^{*}+3$ ). In the case of A2933, $R$ band magnitudes from the APM catalog were used to compute density maps without color selection.

Spectroscopy was performed with EFOSC2 using the grism \#03, which covers the spectral range 3050-6010 $\AA$, with a $F W H M$ of $7.5 \AA$. In general, for each pointing we obtained $2 \times 45$ min exposures. Wavelength calibration was performed in real time, taking spectra of arc calibration lamps (heliumargon) after each exposure. Data reduction was performed using our dedicated IRAF package speXtra for automatic extraction and wavelength calibration of spectra. Radial velocities were obtained using the cross-correlation technique (Tonry \& Davis 1981) with the rvsao package. Cross-correlation was performed with velocity standards observed with the same instrumental configuration during the observing run. The typical velocity error is $\sim 50 \mathrm{~km} \mathrm{~s}^{-1}$, an estimate that we could confirm by performing a cross-check against the data available in the literature (see Sect. 4.3).

The spectroscopic catalogs resulting from our observations are listed for the three clusters in Tables 5-7, where columns are as follows: 1) identification number; 2) and 3) right ascension and declination (J2000.0); 4) radial velocity; 5) velocity error for the cross-correlation; 6) $R_{\mathrm{TR}}$ parameter (Tonry \& Davis 1981; when $R_{\mathrm{TR}}>3$, the cross-correlation redshift can be considered as reliable); and 7) quality flag for the redshift (0: high precision, 1: medium precision).

Objects with a velocity within a $\pm 5000 \mathrm{~km} \mathrm{~s}^{-1}$ window centered on the mean velocity were selected as cluster member candidates. Possible interlopers were rejected by the gap technique. The velocity location and scale of the various clusters (and subclusters) were determined with the biweight estimator using the ROSTAT package (Beers et al. 1990).

Ten normality tests (provided by ROSTAT) were applied to the data. The Dip test for unimodality (Hartigan \& Hartigan 1985) was computed with the diptest package implemented by Martin Maechler in the R environment. The $P_{\text {value }}$ was computed in each case according to the value of the Dip test and the number of objects according to the table provided in Hartigan \& Hartigan (1985). The results of this analysis are displayed in Table 2, where columns are as follows: 1) name of the cluster (or subcluster); 2) and 3) right ascension and declination of the brightest galaxy (BCG) of the (sub)cluster; 4) angular radius $\theta$ of the circle in which galaxies were selected for the ROSTAT analysis; 5) and 6) estimation of location and scale with the biweight technique; 7) number of redshifts used in the analysis; 8) radial velocity of the BCG; 9) number of statistical tests excluding Gaussianity at more than $10 \%$ confidence; 10) and 11) value of the Dip test and of its $P$-value; and 12) mean temperature computed within radius $\theta$ and its error.

To test for multi-modality, we tried in each case to fit the entire velocity distribution with a mixture of Gaussian functions. For this purpose, we have used the powerful EMMIX algorithm (McLachlan \& Krishnan 1997; McLachlan et al. 1999). This program is quite flexible and allows a variety of choices in the fitting. In particular, it does not require to introduce any guess for the initial partition of velocities and the form of the covariance matrix. We applied EMMIX to the data, trying to fit the velocity distribution with mixtures of $N_{\mathrm{g}}$ Gaussian distributions, with $N_{\mathrm{g}}$ varying from 1 to 5 . We retained the best fit on the basis of the $P$-value, and in the case of identical values of $P$-value, on the criterion of the smallest partition number. When a mixture of Gaussians had been fitted to the data, we recomputed the value of the location and scale of each partition with ROSTAT. 
Table 2. Characteristics of the clusters and subclusters.

\begin{tabular}{|c|c|c|c|c|c|c|c|c|c|c|c|}
\hline Name & $\mathrm{RA}(\mathrm{BCG})$ & $\operatorname{Dec}(\mathrm{BCG})$ & $\theta_{\mathrm{p}}$ & $C_{\mathrm{BI}}\left(\mathrm{km} \mathrm{s}^{-1}\right)$ & $S_{\mathrm{BI}}\left(\mathrm{km} \mathrm{s}^{-1}\right)$ & Nobj & $V_{\mathrm{BCG}}\left(\mathrm{km} \mathrm{s}^{-1}\right)$ & Ntest & Dip Test & $P_{\text {value }}$ & $T_{\mathrm{X}}(\mathrm{KeV})$ \\
\hline A2933 & - & - & 15 & $27281 \pm 103$ & $682 \pm 65$ & 47 & - & 0 & 0.0340 & 0.02 & 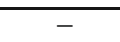 \\
\hline A2933N & 014035.520 & -543054.00 & 3.5 & $26919 \pm 131$ & $578 \pm 70$ & 24 & $27424 \pm 100$ & 0 & 0.056 & 0.12 & $2.83_{-0.26}^{0.31}$ \\
\hline A2933S & 014059.520 & -543726.40 & 3.5 & $27648 \pm 166$ & $568 \pm 142$ & 17 & $28028 \pm 100$ & 0 & 0.071 & 0.15 & $2.80_{-0.17}^{0.1 .9^{6}}$ \\
\hline A2440 & - & - & 15 & $27251 \pm 93$ & $940 \pm 70$ & 103 & - & 0 & 0.022 & 0.01 & - \\
\hline A2440A & 222413.663 & -013134.34 & 2.5 & $28005 \pm 75$ & $178 \pm 90$ & 10 & $27925 \pm 100$ & 10 & 0.067 & 0.03 & -- \\
\hline A $2440 B$ & 222356.941 & -013459.78 & 2.5 & $27212 \pm 195$ & $946 \pm 95$ & 29 & $27032 \pm 16$ & 1 & 0.050 & 0.1 & $4.07_{-0.19}^{0.20}$ \\
\hline A2440C & 222347.814 & -013901.14 & 2.5 & $27234 \pm 188$ & $949 \pm 150$ & 25 & $26816 \pm 19$ & 0 & 0.059 & 0.15 & $4.57_{-0.20}^{0.26}$ \\
\hline A2384 & - & - & 15 & $28263 \pm 154$ & $1114 \pm 106$ & 56 &  & 0 & 0.038 & 0.1 & - \\
\hline A $2384 \mathrm{~N}$ & 215221.962 & -193248.65 & 5 & $28139 \pm 182$ & $1176 \pm 120$ & 44 & $27602 \pm 55$ & 0 & 0.043 & 0.1 & $4.05_{-0.19}^{0.20}$ \\
\hline A2384S & 215209.556 & -194323.71 & 5 & $28814 \pm 241$ & $877 \pm 290$ & 11 & $28696 \pm 53$ & 0 & 0.066 & 0.03 & $4.02_{-0.26}^{0.28}$ \\
\hline
\end{tabular}

Notes. Location, scale, and X-ray mean temperatures of the different subclusters are computed in circles of $\theta_{\mathrm{p}}$ arcmin centered on the position of the BCG (associated with the corresponding X-ray maximum). The measured velocity of the BCG is also listed.
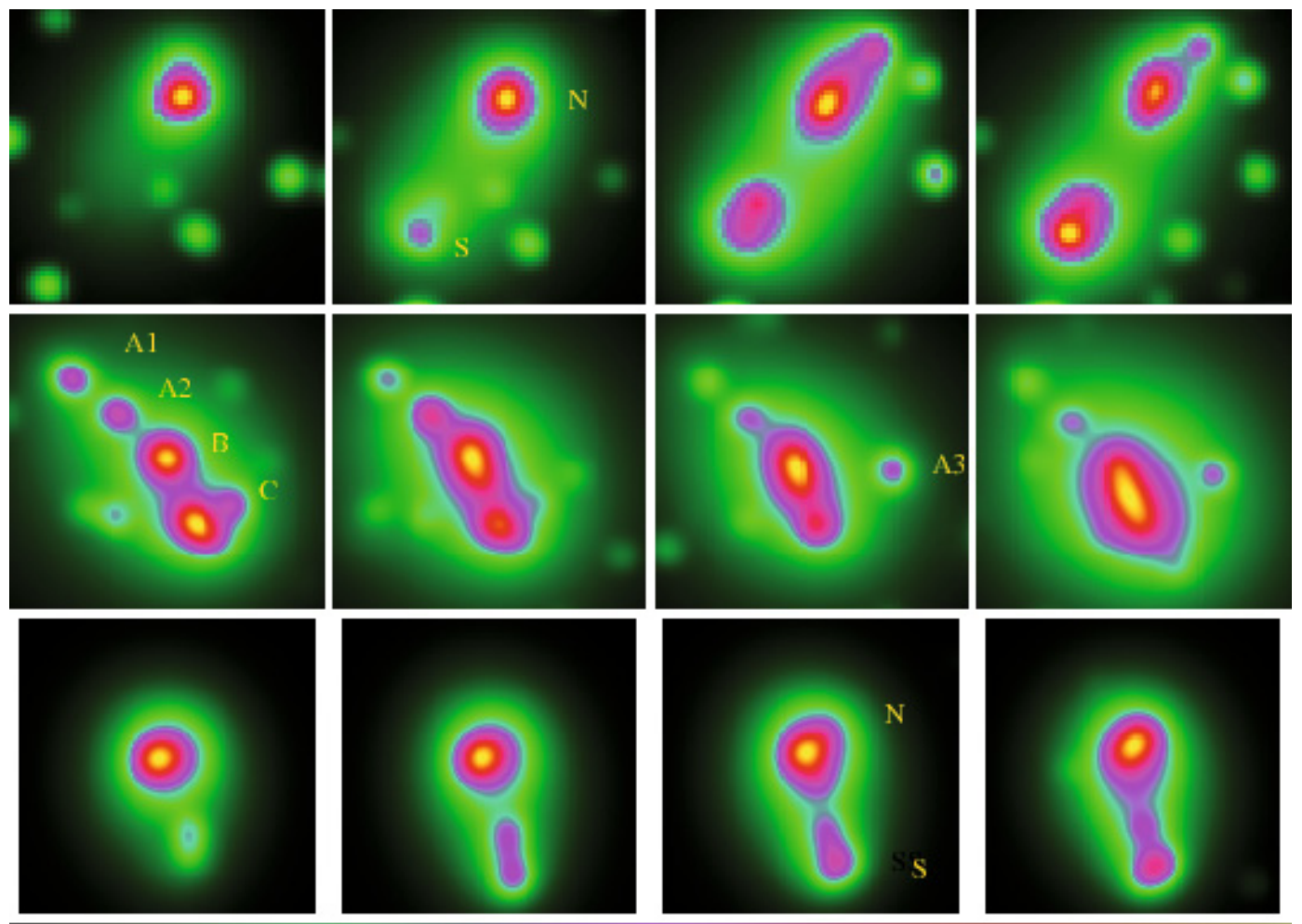

Fig. 1. Galaxy density maps as a function of galaxy luminosity. From top to bottom: A2933, A2440 and A2384. The field of view displayed is $15^{\prime} \times 15^{\prime}$ for A2933 and A2440, and 30' $\times 30^{\prime}$ for A2384. From left to right, magnitude cuts are: $R<18, R<19, R<20$ and $R<21$. The density maps of A2440 and A2384 have been computed for red sequence galaxies.

\section{A2933}

\subsection{X-ray gas morphology and thermal structures}

In X-rays, A2933 is a well defined bimodal system. In Fig. 2 we show the temperature map of A2933 with the X-ray brightness contours superimposed. Looking at the low energy contours, both components look quite regular and have similar brightnesses. We clearly detect the interaction zone as a hot region located in-between the two maxima. The northern unit (A2933N) is slightly elongated along the east-west direction, while the southern unit (A2933S) is orientated along the general NWSE direction connecting the two units. In the temperature map, the most prominent feature is the hot region between the two units, which is statistically significant, because the exposure time of our XMM/EPIC observation was specifically adapted to ensure good statistics in the region of interaction. The temperature distribution of A2933N appears to be quite elongated, while A2933S looks more regular.

\subsection{Galaxy density distribution}

The morphology of Abell 2933 has been very poorly studied at optical wavelengths. In the following analysis, projected density maps were inferred in the field of the cluster from galaxy positions and magnitudes in the APM catalog. 


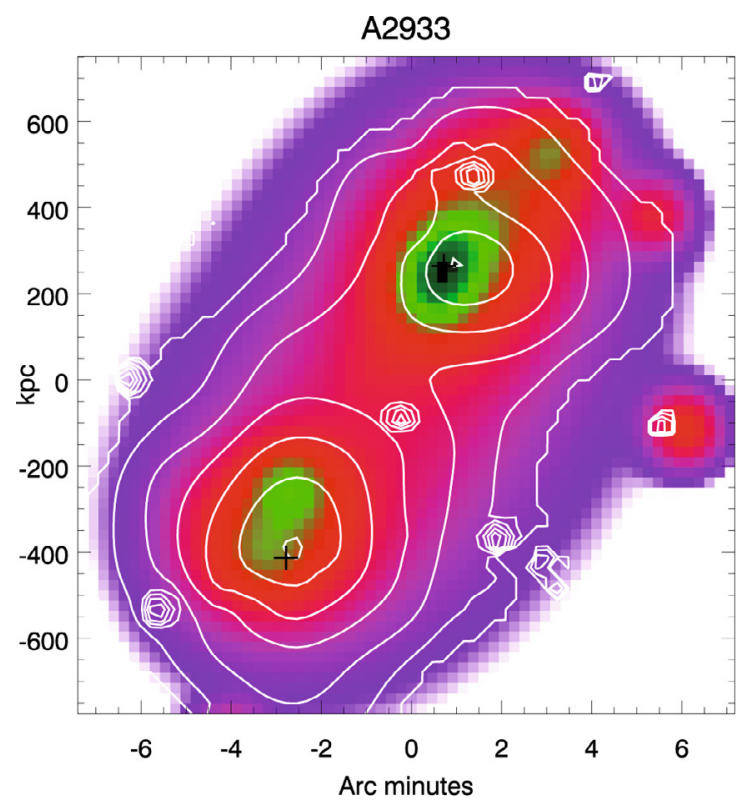

R.A.(0) $014041.23 \operatorname{Dec}(0)-543334.0$

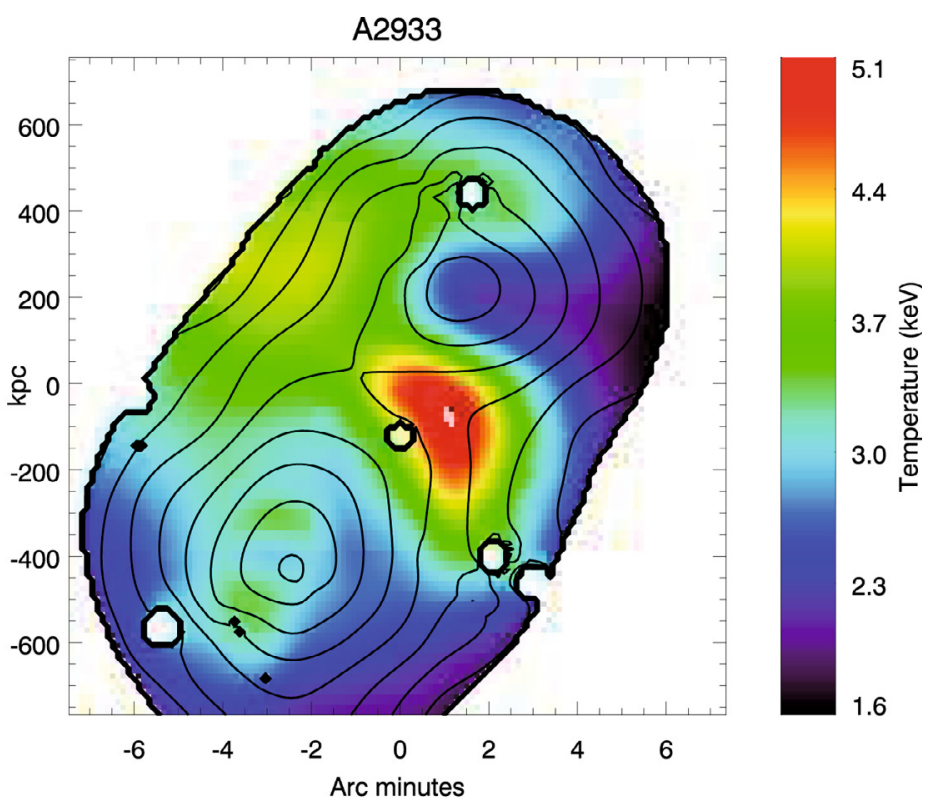

R.A.(0) $014041.99 \operatorname{Dec}(0)-543307.0$

Fig. 2. Left: galaxy density map of A2933 (magnitude cut: $R<20$ ) overlaid on the X-ray luminosity contours (EPIC-XMM counts in the $0.5-2.5 \mathrm{keV}$ band corrected for background and vignetting). The black crosses indicate the BCG positions. Right: ICM temperature map of A2933 overlaid on the X-ray luminosity contours (EPIC-XMM data analyzed by means of wavelet spectral-imaging; see Bourdin et al. 2004, 2008 , for details).

The galaxy density distribution shows a bimodal structure, with two subclusters located on a NW/SE axis at a separation of $\sim 7$ arcmin (Fig. 1). The relative importance of the two components varies with the magnitude cut-off. Selecting bright galaxies $(R<18)$, the NW subcluster (A2933N) is dominant. When including fainter objects, the SE (A2933S) subcluster becomes progressively more prominent (Fig. 1). At faint magnitudes, the NW subcluster shows an extended tail in the NW direction. In Fig. 3, we also show the isodensity contours corresponding to $R<20$, superimposed on the DSS image of A2933. A2933N hosts a couple of very bright galaxies, and is centered on a BCG with a very close companion, while A2933S hosts a single bright elliptical galaxy. One can also note a small density clump at the southern extremity of A2933S. There is a general alignment of the two BCGs, the two subclusters, and the global cluster structure along the NW/SE direction joining A2933N and A2933S.

\subsection{Galaxy velocity distribution}

A2933 was included in the Southern Abell Redshift Survey (SARS, Way et al. 2005), and on the basis of 53 redshifts Muriel et al. (2002) estimated the cluster global velocity and velocity dispersion $\left(27709 \pm 105 \mathrm{~km} \mathrm{~s}^{-1}\right.$ and $759 \pm 72 \mathrm{~km} \mathrm{~s}^{-1}$, respectively); they also showed that the velocity dispersion as a function of radius appears to be constant to $5 \mathrm{Mpc} / h$ from the cluster center. Their data, however, sample the cluster and its environment on large scales and at relatively bright magnitudes. Since we are interested in the merging process, we observed the central region including the two main clumps at deeper magnitudes.

We measured 71 redshifts in the $30^{\prime} \times 30^{\prime}$ field of A2933, listed in Table 5, from which 47 objects are identified as cluster members. We have only 11 redshifts (among 53) in common with them, because of the different region sampled. Excluding one discrepant case, the redshifts of common objects are all in excellent agreement, with a mean velocity difference of $14 \mathrm{~km} \mathrm{~s}^{-1}$ and a standard deviation of $59 \mathrm{~km} \mathrm{~s}^{-1}$. From our redshift sample, we analyzed the velocity distribution in the central region of A2933. We measured a location of $C_{\mathrm{BI}}=$ $27281 \pm 103 \mathrm{~km} \mathrm{~s}^{-1}$ and a scale of $S_{\mathrm{BI}}=682 \pm 65 \mathrm{~km} \mathrm{~s}^{-1}$ (Table 2). Our estimate of velocity location is significantly lower $\left(\sim 400 \mathrm{~km} \mathrm{~s}^{-1}\right)$ than that found by Muriel et al. (2002). These differences may be caused by variations in sampling. Their sample covers a much larger area, that is not centered on the two subclusters but is shifted to the south, which we show to be a region of higher mean velocity. We checked that their estimate of mean velocity and our estimate for southern component (see below) are consistent.

The velocity histogram (plotted in Fig. 4) clearly shows a multi-modal distribution, with a sharp peak at $\sim 26200 \mathrm{~km} \mathrm{~s}^{-1}$, and a more dispersed structure, with two wider peaks at $\sim 26800 \mathrm{~km} \mathrm{~s}^{-1}$ and $\sim 27500 \mathrm{~km} \mathrm{~s}^{-1}$. In Fig. 3, cluster members are indicated by different symbols and colors for galaxies with velocities within each of the three peaks in the histogram. Most of the galaxies with velocities located in the sharp peak at $\sim 26200 \mathrm{~km} \mathrm{~s}^{-1}$ (purple squares) are located in A2933N. Moreover, the region of A2933S, including its southern extension, is populated mostly by galaxies in the highest velocity peak at $\sim 27500 \mathrm{~km} \mathrm{~s}^{-1}$, while objects with velocities within the peak at $\sim 26800 \mathrm{~km} \mathrm{~s}^{-1}$ are mainly in the A2933N region.

To quantitatively assess the significance of the multimodality in the velocity distribution suggested by the histogram, we applied our set of statistical tests (running a set of tests is useful because they have different sensitivities to subclustering; see Pinkney et al. 1996). Ten out of 10 of the normality tests provided by ROSTAT do not find significant deviations from Gaussianity (see Table 2). However, the Dip-test excludes unimodality at more than $2 \%$ significance.

We finally applied EMMIX, where the data were fitted without a priori constraints on the covariance matrix. A three Gaussian mixture inferred a $P$-value of 0.03 , indicating that the Gaussian hypothesis is excluded at a very high significance level. This mixture fits the main structure with a Gaussian of 


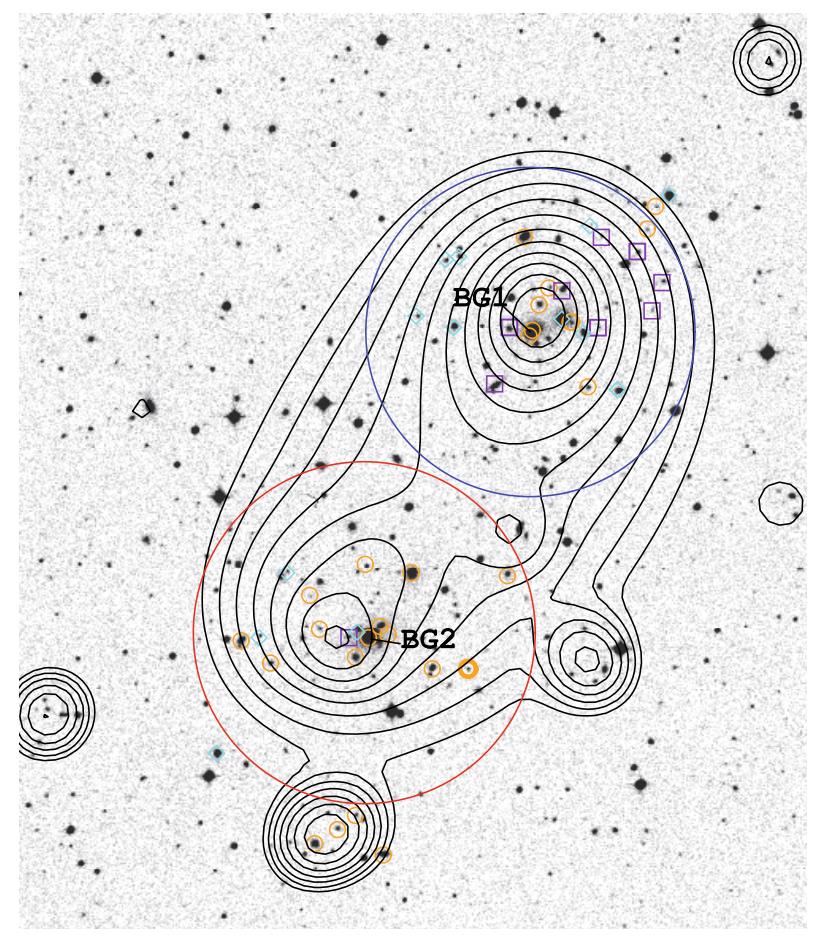

Fig. 3. DSS region $\left(17^{\prime} \times 20^{\prime}\right)$ centered on A2933 (North is up and East is to the left). Galaxies identified as cluster members from spectroscopy are marked with different symbols corresponding to the three major peaks in the velocity histogram (purple squares: [26000, $26500] \mathrm{km} \mathrm{s}^{-1}$, cyan diamonds: [26500, 27200$] \mathrm{km} \mathrm{s}^{-1}$, orange circles: [27 200, 28500$] \mathrm{km} \mathrm{s}^{-1}$ ) which is plotted in Fig. 4. The isocontours corresponding to the galaxy density map with $R<19$ are superimposed. The velocity histograms for the galaxies in the two circles (which have a 3.5 arcmin radius and are centered on the two subclusters) are displayed in Fig. 5.

Table 3. Results of the mixture of Gaussian with EMMIX.

\begin{tabular}{lccccc}
\hline \hline Name & part & $v 1\left(\mathrm{~km} \mathrm{~s}^{-1}\right)$ & $S_{\mathrm{BI}}\left(\mathrm{km} \mathrm{s}^{-1}\right)$ & $N_{\mathrm{g}}$ & Allocation rate \\
\hline $\mathrm{A} 2933$ & 1 & $27364 \pm 84$ & $501 \pm 45$ & 39 & 0.984 \\
$\mathrm{~A} 2933$ & 2 & $26145 \pm 27$ & $65 \pm 25$ & 6 & 0.999 \\
$\mathrm{~A} 2933$ & 3 & $28873 \pm-$ & - & 2 & 1.0 \\
\hline A2440 & 1 & $27080 \pm 96$ & $889 \pm 62$ & 96 & 0.95 \\
$\mathrm{~A} 2440$ & 2 & $28058 \pm 25$ & $33 \pm 25$ & 5 & 0.30 \\
$\mathrm{~A} 2440$ & 3 & $30360 \pm-$ & - & 2 & 1.0 \\
\hline $\mathrm{A} 2384$ & 1 & $29760 \pm 122$ & $365 \pm 75$ & 13 & 0.968 \\
$\mathrm{~A} 2384$ & 2 & $28546 \pm 55$ & $200 \pm 27$ & 20 & 0.974 \\
$\mathrm{~A} 2384$ & 3 & $27312 \pm 168$ & $654 \pm 135$ & 23 & 0.833 \\
\hline
\end{tabular}

Notes. Errors have been estimated with ROSTAT.

mean $V=27364 \mathrm{~km} \mathrm{~s}^{-1}$ and dispersion $\sigma=501 \mathrm{~km} \mathrm{~s}^{-1}$ (partition 1), the sharp peak at lower velocity with a Gaussian having mean $V \sim 26145 \mathrm{~km} \mathrm{~s}^{-1}$ and a very low dispersion $\sigma=$ $65 \mathrm{~km} \mathrm{~s}^{-1}$ (partition 2), and the small excess at high velocities (partition 3). Results are listed in Table 3. The sharp low velocity excess consists of six galaxies: all but one are located in the northern subcluster (purple squares in Fig. 3). These galaxies are likely to form a low velocity group probably infalling into A2933N. In the following, we exclude partition 3 (with only 2 objects) from the analysis. No significant results are obtained with EMMIX when fitting with mixture with a higher partitioning to the data.

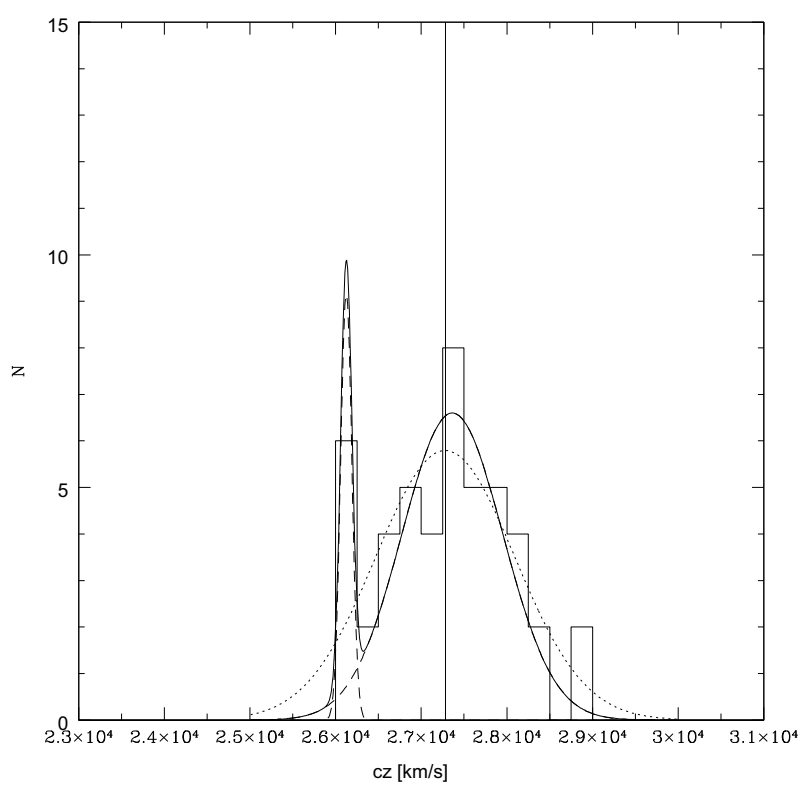

Fig. 4. Velocity histogram of A2933 (binning of $250 \mathrm{~km} \mathrm{~s}^{-1}$ ). The best Gaussian fit for the whole distribution (dotted line) is centered on the vertical solid line which gives the location value. Location and scale of the Gaussian (i.e. mean velocity and velocity dispersion) were estimated with ROSTAT. We also show the two Gaussian functions corresponding to partition 1 and 2 of the best mixture 3 partitions fit by EMMIX (dashed lines) and the composite function (solid line).

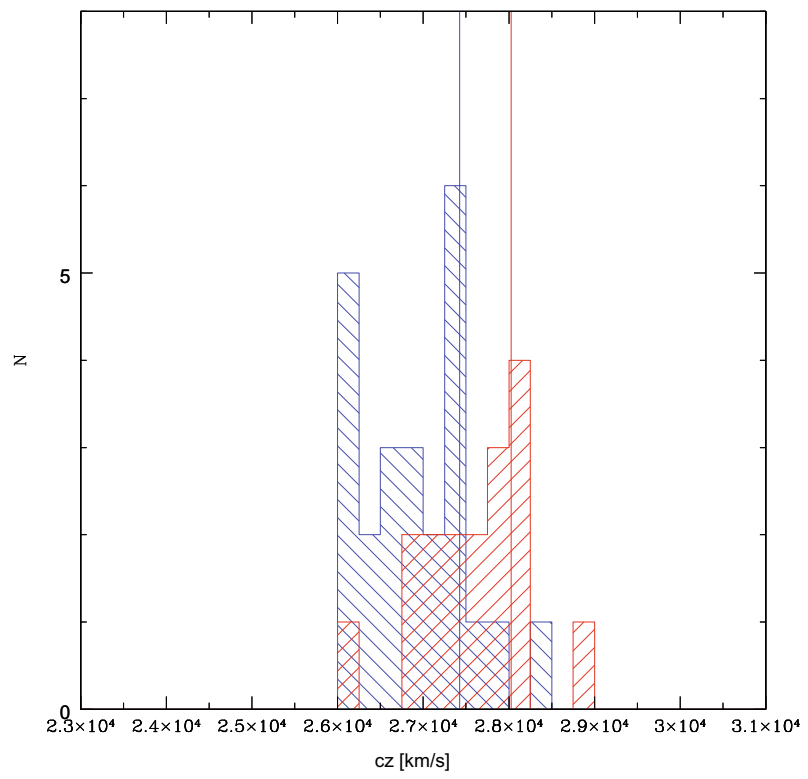

Fig. 5. Velocity histograms of A2933N (blue) and A2933S (red). For each subcluster we included galaxies within a radius of $3.5^{\prime}$ from its X-ray center. Solid lines: velocities of the brightest galaxy in the Northern (blue) and Southern (red) subclusters.

\subsection{X-ray/optical combined analysis}

The projected density maps of A2933 for galaxies and for gas provide similar views of the cluster (Fig. 2): the X-ray and optical isocontours of the NW and SE subclusters, A2933N and A2933S, closely correspond and are centered on the cold cores in the temperature map (Fig. 2). The X-ray contours are roughly centered on the BCGs for both the NW and SE subclusters. These results suggest that the system is in a pre-merger phase. 
However, in the case of the NW subcluster, the BCG seems slightly offset eastwards with respect to the X-ray centroid. The inner X-ray isocontours and the temperature map are elongated along an E-W axis while the outer isocontours are orientated along the general SE-NW axis also connecting the two brightest galaxies of A2933N.

As the density distribution of galaxies shows a clear bimodal behavior, we analyzed separately the velocity distributions of A2933N and A2933S, selecting the galaxies within a radius of 3.5' from each subcluster center (see Fig. 3). The corresponding histograms are displayed in Fig. 5. The velocity distributions of the two subclusters are offset from each other, the NW subcluster lying at lower velocities than the SE subcluster, but there is significant overlap. Moreover, they are quite different. A2933N has a multi-peak structure with the sharp excess at low velocities previously identified with EMMIX, while A2933S has a more continuous distribution with an extended low velocity tail that falls in the velocity range of A2933N.

Departure from normality was tested when analyzing both subclusters individually, and neither the normality tests nor the Dip test allowed us to significantly exclude the hypothesis of a Gaussian velocity distribution. The ROSTAT analysis of the two velocity distributions shows that the scales (velocity dispersions) are comparable for both subclusters. These scales are in good agreement with those expected from the measured X-ray temperatures, assuming the typical scaling relation between $\sigma$ and $k T$ (Lubin \& Bahcall 1993; Girardi et al. 1998; Wu et al. 1998, see Fig. 6), suggesting that gas and galaxies are in equilibrium.

The dynamical analysis also confirms a significant offset for locations (mean velocities) of $\sim 730 \pm 210 \mathrm{~km} \mathrm{~s}^{-1}$ : A2933S has a significantly higher mean velocity than A2933N $\left(\sim 27648 \mathrm{~km} \mathrm{~s}^{-1}\right.$ vs. $\left.\sim 26919 \mathrm{~km} \mathrm{~s}^{-1}\right)$. This trend is also followed by the velocities of the brightest cluster members: the BCG in A2933N (BCG1) and its close companion have comparable velocities $\left(27350 \mathrm{~km} \mathrm{~s}^{-1}\right.$ and $\left.27424 \mathrm{~km} \mathrm{~s}^{-1}\right)$; the second brightest galaxy has a lower velocity $\left(26951 \mathrm{~km} \mathrm{~s}^{-1}\right)$, while the brightest galaxy in A2933S (BCG2) has a higher velocity $\left(28028 \mathrm{~km} \mathrm{~s}^{-1}\right)$. This implies that there is a significant offset of $\sim 600 \pm 140 \mathrm{~km} \mathrm{~s}^{-1}$ between BCG1 and BCG2.

The velocities of the two BCGs also differ from the mean velocity of their host subcluster by $505 \pm 165 \mathrm{~km} \mathrm{~s}^{-1}$ and $380 \pm$ $200 \mathrm{~km} \mathrm{~s}^{-1}$ for A2933N and A2933S, respectively, which is statistically significant for A2933N.

However, they coincide with the locations of the highest peaks in the velocity histograms of their respective subclusters A2933N and A2933S (excluding the sharp peak at $\sim 26200 \mathrm{~km} \mathrm{~s}^{-1}$ ), and these peaks are slightly shifted from the mean velocity of each subcluster, due to the skewness of the velocity distributions (Fig. 5). All these results suggest that the velocity distributions have started to mix with each other, but that the structure of the two subclusters is still not strongly affected; this implies that the clusters have not yet crossed each other.

Therefore the combined X-ray-optical analysis indicates that A2933N and A2933S are in an advanced pre-merger stage. The presence of two very bright galaxies along an NE-SW axis, the elongations of the inner X-ray contours and the temperature map along the E-W direction of A2933N, and the existence of a distinct low velocity, low dispersion velocity component, suggest that $\mathrm{A} 2933 \mathrm{~N}$ has also undergone some previous merging and is still accreting a small group.

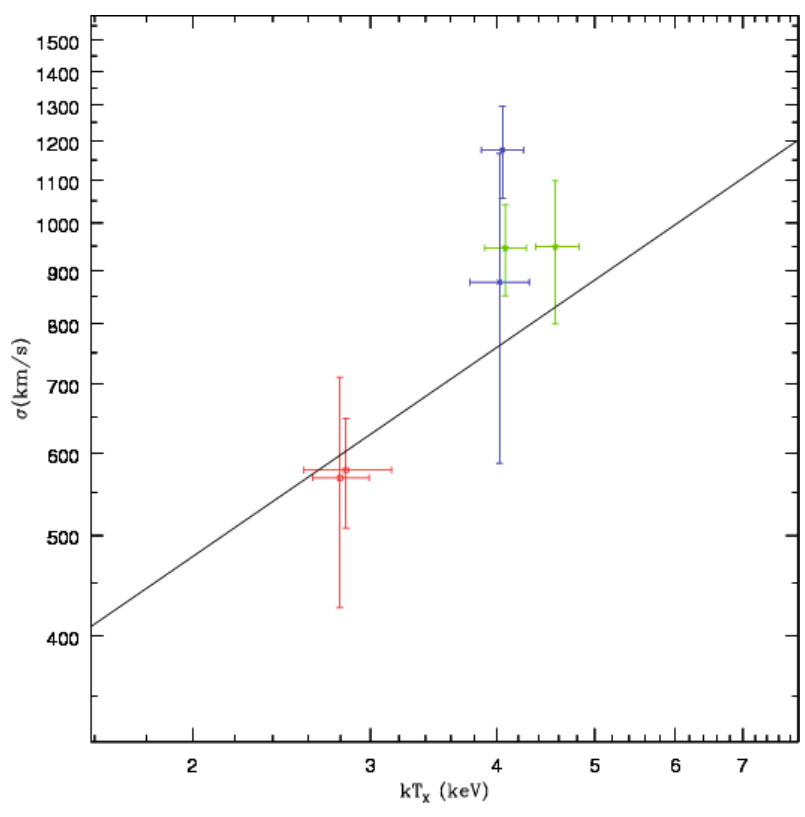

Fig. 6. The $\sigma-T_{\mathrm{X}}$ relation for the subclusters A2933N and A2933S (red), A2440B and A2440C (green), A2384N and A2384S(blue). The straight line is the relation $\sigma=10^{2.47 \pm 0.08} T^{0.67 \pm 0.09}$ (Wu et al. 1998).

\subsection{Dynamical analysis}

We now provide mass estimates for the subclusters and the total mass of A2933 (analogous sections are devoted to A2440 and A2384). For each system, we discuss the uncertainties in the mass estimates. An obvious uncertainty is related to the assumption of dynamical or hydrostatic equilibrium. For example, velocity dispersions might not simply reflect the subcluster potential and might be overestimated. Another uncertainty is due to the limited field in which it is possible to estimate the harmonic radius: the circle within which we estimate this radius cannot intersect the nearby subcluster(s). As a consequence, the virial radius might be underestimated. The mass may also be underestimated due to the pressure term.

\subsubsection{Mass estimates}

From the previous analysis, A2933 is very likely in a pre-merger stage, in which subclusters begin to interact but cores have not yet crossed each other. We may then assume that the states of the two subclusters do not strongly deviate from dynamical equilibrium, so that the virial theorem and the hydrostatic equilibrium can be safely applied to determine optical and X-ray mass estimates. These estimates will be used in the following to constrain the parameters of the collision by means of a two-body analysis.

In the optical, we estimated the harmonic radius of the two subclusters from the projected distribution of all galaxies with $R<20$ and within a radius of 3.5 arcmin from the BCG; from the harmonic radius we derived the virial radius and, in combination with the velocity dispersion, we estimated the virial mass. Finally, extrapolating the density profile, we estimated the mass $M_{200}$ corresponding to a density contrast $\bar{\rho} / \rho_{\mathrm{c}}=200$ (for more details see e.g. Maurogordato et al. 2008). We find for $\mathrm{A} 2933 \mathrm{~N} M_{200}=2.0 \pm 0.4 \times 10^{14} M_{\odot}$ and for A2933S $M_{200}=$ $(2.2 \pm 0.4) \times 10^{14} M_{\odot}$, with a ratio $M_{\mathrm{A} 2933 \mathrm{~S}} / M_{\mathrm{A} 2933 \mathrm{~N}}=1.1 \pm 0.6$.

We can also estimate the mass ratio using the X-ray scaling relations. The mean X-ray temperatures of A2933N and A2933S 
are respectively $k T_{\mathrm{A} 2933 \mathrm{~N}}=2.11 \pm 0.25 \mathrm{keV}$ and $k T_{\mathrm{A} 2933 \mathrm{~S}}=2.7 \pm$ $0.2 \mathrm{keV}$. Assuming $M \propto T_{\mathrm{X}}^{3 / 2}$, we obtain a mass ratio between the two components around 1.5:1.

On the other hand, when we estimate masses using the brightness and temperature profiles of each unit, assuming hydrostatic equilibrium for each subcluster, we find that $M_{\mathrm{A} 2933 \mathrm{~N}}=2.6 \pm 0.4 \times 10^{14} M_{\odot}$ and $M_{\mathrm{A} 2933 \mathrm{~S}}=2.76 \pm 1.5 \times 10^{14} M_{\odot}$ leading to a mass ratio of $\frac{M_{\mathrm{A} 2933 \mathrm{~S}}}{M_{\mathrm{A} 2933 \mathrm{~N}}}=1.2 \pm 0.6$.

While the exact values could be systematically affected by deviations from equilibrium, there is a good consistency between the optical and X-ray mass estimates, and we can conclude that the two subclusters have comparable masses, with a total mass for A2933 of $M_{200} \sim 5 \times 10^{14} M_{\odot}$.

\subsubsection{Two-body analysis}

We applied the two-body dynamical formalism (Beers et al. 1982; Gregory \& Thomson 1984) to the two subclusters in A2933. One of the variants of this method establishes a relationship between the total mass $M$ of the bimodal cluster and the angle $\alpha$ between the plane of the sky and the collision axis of the two clumps (Barrena et al. 2002; Ferrari et al. 2005). The input parameters derived from the observations are the relative radial velocity between the two subclusters $V_{\mathrm{r}}$ and their projected spatial separation $R_{\mathrm{p}}$. For these observables, we chose to take the values of the projected spatial separation and the radial relative velocity (at rest) between the two BCGs $\left(R_{\mathrm{p}}=0.75 \mathrm{Mpc}\right.$ and $V_{\mathrm{r}}=550 \mathrm{~km} \mathrm{~s}^{-1}$ ), which are more clearly defined than that of the subclusters. We obtained similar results when using values from the subcluster centroids. In addition, the formalism requires us to define the time $t_{0}$ that has elapsed since the epoch of the last encounter between the two subclusters. We tested different scenarios, including a pre-merging scenario $\left(t_{0} \sim 12.2 \mathrm{Gyr}\right.$, i.e. the age of the Universe at the redshift of A2933, implying that the two systems have never yet crossed each other), a recent $\left(t_{0}=0.5 \mathrm{Gyr}\right)$ and an older $\left(t_{0}=1.0 \mathrm{Gyr}\right)$ post-merger event. Considering the total mass of the cluster derived from optical observations $\left(5 \times 10^{14} M_{\odot}\right)$, the post-merger cases provide only one possible bound outgoing solution (i.e. the two subclusters are moving apart). In the pre-merger case, Fig. 7 reveals two possible bound incoming solutions, the first with $\alpha \sim 15^{\circ}$ (BIa) and the second with $\alpha \sim 75^{\circ}$ (BIb), and a bound outgoing solution (BO). For BIa, the "real" separation between the two subclusters appears to be small $(\sim 0.8 \mathrm{Mpc})$ and the relative velocity high $\left(V \sim 2000 \mathrm{~km} \mathrm{~s}^{-1}\right)$, while for BIb the two components appears to be more distant $(\sim 3.5 \mathrm{Mpc})$ and the relative velocity comparable to the projected observed one $\left(V \sim 560 \mathrm{~km} \mathrm{~s}^{-1}\right)$. The results are listed in Table 4.

\subsection{The scenario for $A 2933$}

The combined X-ray optical analysis has shown: a) two subclusters, visible in both X-ray and optical maps, and associated with cool cores detected in the temperature maps; b) a hot region in between the two subclusters; c) a perturbed velocity distribution, showing a velocity offset (at rest) of $\sim 700 \mathrm{~km} \mathrm{~s}^{-1}$ between the two subclusters. This suggests that the interaction between the two subclusters is in its initial phase. This lead us to exclude the post-merger cases in the two-body analysis, and to examine the pre-merger one. Solutions with large values of $\alpha$ : BIb, and UO are quite improbable since they would imply large spatial separations between the subclusters $(\sim 3.5 \mathrm{Mpc}$ and $11 \mathrm{Mpc}$, respectively), which is unlikely taken into account the clear signs

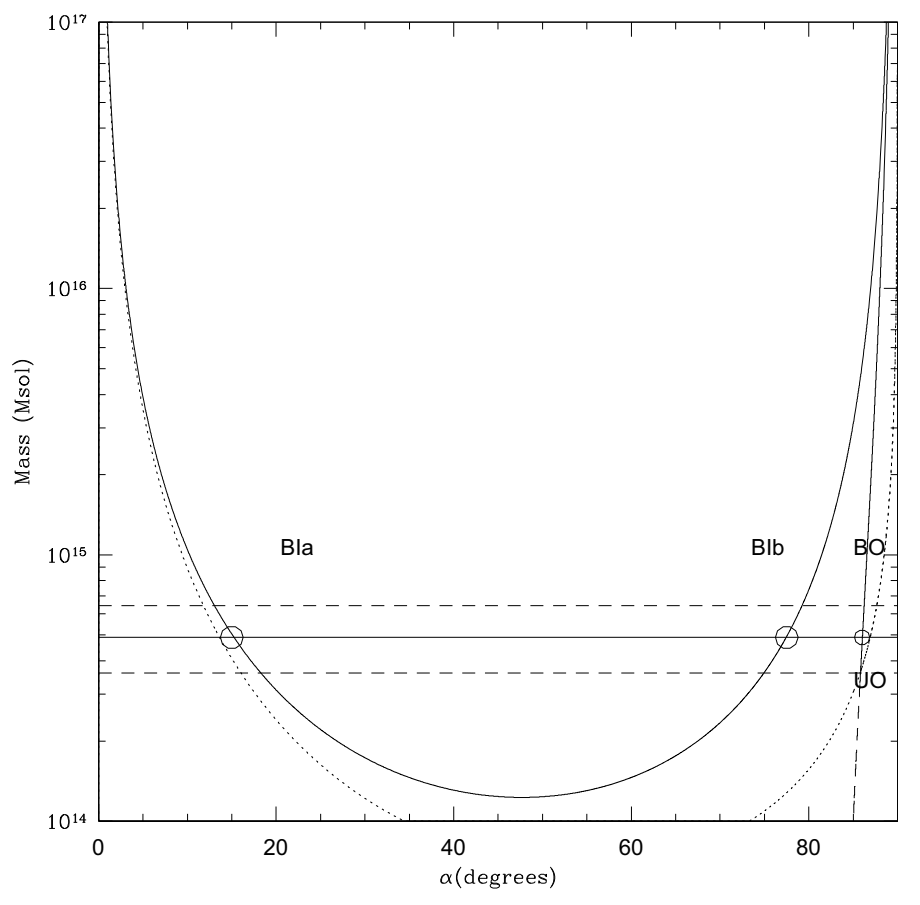

Fig. 7. The sum of the virial masses of A2933N and A2933S as a function of the projection angle $\alpha$. The horizontal lines show the mass estimate (full line) and errors (dashed lines) (see Sect. 4.5.1). The projected distance of $0.75 \mathrm{Mpc}$ and radial velocity difference at rest of $550 \mathrm{~km} \mathrm{~s}^{-1}$ are derived from the two BCG's location and velocities. The two systems were at zero separation $12.2 \mathrm{Gyr}$ ago. The dotted line represents the Newtonian criterion for gravitational binding. Two bound incoming (BIa and $\mathrm{BIb}$ ) and one bound outgoing (BO) are found compatible with our mass estimates.

of interaction between the two merging units. The most likely solution for A2933 is thus (BIa), i.e. a two-body pre-merger nearly on the plane of the sky $\left(\alpha \sim 15^{\circ}\right)$. The high relative velocity $\left(\sim 2000 \mathrm{~km} \mathrm{~s}^{-1}\right)$ between the two components implied by this solution is a common feature in merging clusters.

\section{A2440}

Mohr et al. (1996) performed a combined X-ray and optical analysis of A2440, based on optical imaging and spectroscopy (48 redshifts), and Einstein X-ray data. Our work significantly extends this study, being based on a larger spectroscopic sample (more than double the total number of redshifts) and new X-ray data, including the gas temperature maps.

\subsection{X-ray gas morphology and thermal structures}

The X-ray observations detect a gas emission with an elongated structure connecting two brightness peaks (B) and (C) to a northern and a less luminous component (A) (Fig. 8). The two brightness peaks are associated with cool cores $(k T \simeq 2.8-3.5 \mathrm{keV})$, and are surrounded by hotter gas $(k T \simeq 5 \mathrm{keV})$; a significant estimate of the temperature of the (A) component is not possible due to the strong contamination from the innermost cluster regions.

The southern emission peak (C) is delimited by gas brightness and temperature jumps across the white sector shown in Fig. 8. As discussed above, these discontinuities are signatures of a cold front delimiting the edge of the southern cold core.

To investigate the nature of the gas density jump observable across the white sector in Fig. 8, we model the gas 3D structure 
S. Maurogordato et al.: Merging history of three bimodal clusters

Table 4. Two-body model solutions for the A2933N-A2933S, A2440A-A2440(B+C), and A2384N-A2384S systems.

\begin{tabular}{ccccccc}
\hline \hline Cluster & \multirow{2}{*}{$t_{0}$} & Solution & $\alpha(\mathrm{deg})$ & $R(\mathrm{Mpc})$ & $R_{\mathrm{m}}(\mathrm{Mpc})$ & $V\left(\mathrm{~km} \mathrm{~s}^{-1}\right)$ \\
\hline $\mathrm{A} 2933 \mathrm{~N} / \mathrm{S}$ & $12.2 \mathrm{Gyr}$ & BIa & 15 & 0.8 & 4.1 & 2120 \\
& & BIb & 78 & 3.5 & 4.7 & 560 \\
& & BO & 86 & 10.9 & 47. & 551 \\
\hline $\mathrm{A} 2933 \mathrm{~N} / \mathrm{S}$ & $0.5 \mathrm{Gyr}$ & $\mathrm{BO}$ & 47 & 1.1 & 1.5 & 1055 \\
\hline $\mathrm{A} 2933 \mathrm{~N} / \mathrm{S}$ & $1.0 \mathrm{Gyr}$ & $\mathrm{BO}$ & 65 & 1.8 & 2.5 & 862 \\
\hline $\mathrm{A} 2440 \mathrm{~A} / \mathrm{B}+\mathrm{C}$ & $12.2 \mathrm{Gyr}$ & $\mathrm{BIa}$ & 11.37 & 0.61 & 5.3 & 3700 \\
\hline & & BIb & 82.4 & 4.5 & 6.1 & 740 \\
\hline & & BO & 87.5 & 14.3 & 75 & 730 \\
\hline $\mathrm{A} 2384 \mathrm{~N} / \mathrm{S}$ & $12.2 \mathrm{Gyr}$ & $\mathrm{BIa}$ & 17 & 1.2 & 6.5 & 3440 \\
& & BIb & 77 & 5.0 & 7.3 & 1030 \\
\hline $\mathrm{A} 2384 \mathrm{~N} / \mathrm{S}$ & $0.5 \mathrm{Gyr}$ & $\mathrm{BO}$ & 46 & 1.6 & 2.0 & 1390 \\
\hline $\mathrm{A} 2384 \mathrm{~N} / \mathrm{S}$ & $1.0 \mathrm{Gyr}$ & $\mathrm{BO}$ & 65 & 2.6 & 3.2 & 1110 \\
\hline $\mathrm{A} 2384 \mathrm{~N} / \mathrm{S}$ & $2.0 \mathrm{Gyr}$ & $\mathrm{BO}$ & 75 & 4.4 & 6.0 & 1030 \\
& & BIa & 25 & 1.2 & 2.2 & 2354 \\
& & BIb & 53 & 1.9 & 2.3 & 1240 \\
\hline
\end{tabular}

Notes. Various possibilities are considered for the evolutionary phase: the pre-merger case $\left(t_{0}=12.2\right.$ Gyr), and post-mergers seen at $t_{0}$ after the first passage. For each solution, we derive the angle $\alpha$ between the line connecting the two components and the plane of the sky, the spatial separation of the subclusters $R$, their separation at maximum expansion $R_{\mathrm{m}}$ and their relative velocity $V$.
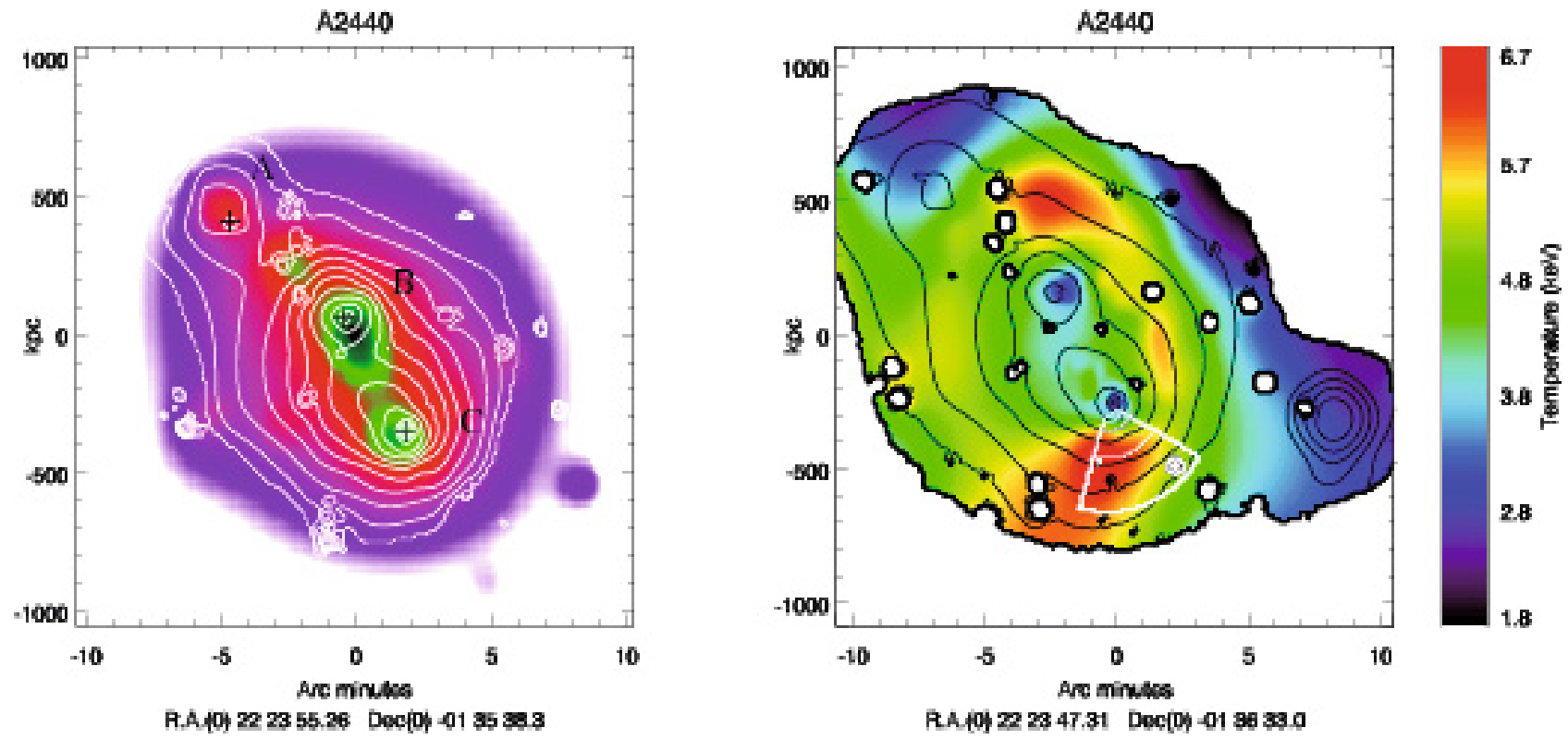

Fig. 8. A2440: left: galaxy density map (mag. cuts: $R<19$ ) overlaid on the X-ray luminosity contours (EPIC-XMM counts in the $.5-2.5 \mathrm{keV}$ band corrected for background and vignetting). Black crosses indicate the BCG positions. Right: ICM temperature map overlaid on the X-ray luminosity contours (EPIC-XMM data analyzed through wavelet spectral-imaging, see Bourdin et al. 2004, 2008, for details).

with disrupted density and temperature profiles (see Eqs. (20) and (21) of Bourdin et al. 2008). As shown in Fig. 10, fitting the slope, jump position, and amplitude of these functions reveals a discontinuity in gas density, $D_{n}=1.82 \pm 0.06$, and $3 \mathrm{D}$ temperature, $D_{\mathrm{T}}=1.76_{-0.06}^{+0.08}$, located at $110 \mathrm{kpc}$ to the south of the cool core. The pressure continuity that is measurable across the jump $\left(D_{\mathrm{P}}=D_{\mathrm{T}} / D_{n}=0.97 \pm 0.05\right)$ identifies the discontinuity as a cold front. As already observed in various interacting systems, this cold front is likely to delimit a stripped cold core moving outwards from the hotter embedding ICM.

\subsection{Galaxy density distribution}

The galaxy density maps computed for RS galaxies for different magnitude cuts (Fig. 1) show that the general structure of
A2440 is strongly elongated along a NE/SW axis and includes multiple clumps. At bright magnitudes $(R<18)$, four groups are detected: the two most significant subclusters $\mathrm{B}$ and $\mathrm{C}$ are identified as the main cluster, and two fainter ones are detected at the NE extent: A1 and A2. When including faint galaxies, the two components corresponding to the main cluster B and $\mathrm{C}$ progressively merge to appear as a single dominant elongated structure $(R<21)$, while the NE groups A1 and A2 are less visible and a new (but faint) component A3 is detected on the western side.

The three brightest galaxies are located near the three density peaks A1, B, and C identified at $R<18$ and $R<19$ (Fig. 11).

There is a strong alignment between the main axis of the bright galaxies, the position angle of the subclusters and the $\mathrm{NE} / \mathrm{SW}$ axis of the whole cluster. 


\subsection{Galaxy velocity distribution}

The first analyses of the dynamics of A2440 were performed by Beers et al. (1991) and Mohr et al. (1996). We now have a much larger dataset: from our EFOSC2 observations, 97 redshifts have been obtained in the $30^{\prime}$ by $30^{\prime}$ field centered on A2440. We merged this catalog with redshifts available in the literature, which led to the final redshift catalog of 150 objects (with 10 twice observed objects). A comparison with 10 measured redshifts available in the literature gives a mean difference of $30 \mathrm{~km} \mathrm{~s}^{-1}$ and a standard deviation of $60 \mathrm{~km} \mathrm{~s}^{-1}$. Restricting this sample to cluster members, we are left with 103 objects. The velocity histogram is shown in Fig. 12.

In Fig. 11, cluster members are indicated by purple squares and orange circles when belonging to one of the two major velocity peaks in the histogram at $\sim 27000 \mathrm{~km} \mathrm{~s}^{-1}$ and $28000 \mathrm{~km} \mathrm{~s}^{-1}$ respectively. One can see that galaxies belonging to both velocity peaks populate subclusters $\mathrm{B}$ and $\mathrm{C}$, while all galaxies in subcluster A1 belong to the highest velocity peak.

Measurements of location and scale of the velocity distribution with the biweight estimator give $C_{\mathrm{BI}}=27251 \pm 93 \mathrm{~km} \mathrm{~s}^{-1}$ and $S_{\mathrm{BI}}=940 \pm 70 \mathrm{~km} \mathrm{~s}^{-1}$. While none of the 10 ROSTAT normality tests identify significant deviations from Gaussianity, the Dip test rejects unimodality at the 1 percent level (Table 2).

EMMIX fits a mixture with two partitions isolating the main component and an excess of two galaxies at $V \sim 28058 \mathrm{~km} \mathrm{~s}^{-1}$ with a very significant $P$-value (0.01). With three partitions, EMMIX isolates the main component with mean velocity $V \sim$ $27080 \mathrm{~km} \mathrm{~s}^{-1}$ and velocity dispersion $\sigma=890 \mathrm{~km} \mathrm{~s}^{-1}$ (partition 1), a very sharp Gaussian $\left(\sigma \sim 33 \mathrm{~km} \mathrm{~s}^{-1}\right)$, corresponding to the previously mentioned excess with mean velocity $V \sim 28058 \mathrm{~km} \mathrm{~s}^{-1}$ (partition 2), and a group of two galaxies at $\sim 30337 \mathrm{~km} \mathrm{~s}^{-1}$ (partition 3), which are not considered in the following. The best-fit Gaussian functions (partition 1 and 2) are plotted in Fig. 12. However, the relatively high value of $P_{\text {value }}$ obtained (0.23) indicates that this three partition fit is less significant than the two partition one. No significant results are obtained with EMMIX when fitting with a larger number of partitions.

\subsection{A2440: X-ray/optical combined analysis}

Examining Fig. 8, where we show the X-ray isocontours and the galaxy projected density distribution, we see that the central $\mathrm{X}$-ray bimodal structure $(\mathrm{B}+\mathrm{C})$ is nearly coincident with the optical bimodal structure $(\mathrm{B}+\mathrm{C})$ in the density map at $R<19$ (and coincident with that at $R<18$ ). The $\mathrm{X}$-ray A component is coincident with the NE subcluster A1 identified in the galaxy density maps, while the subclusters A2 and A3 are not detected in $\mathrm{X}$-rays. The three X-ray maxima A, B, and C are well centered on the three corresponding BCGs (Fig. 8). We have seen that at fainter magnitudes the optical bimodal structure merges into one structure: its density peak is centered between the X-ray maxima B and C. These three subclusters, identified in X-rays and with optical counterparts, are referred to as A2440A, A2440B, and A2440C.

To analyze the velocity distribution in A2440A, A2440B, and A2440C, we defined three subsamples including galaxies within circular regions of radius 2.5 arcmin (the largest compatible with negligible overlap) and centered on the corresponding $\mathrm{X}$-ray maxima, which coincide with the BCGs positions. These circular regions are shown in Fig. 11.

In Fig. 13, we show the corresponding velocity histograms with different colors (blue, green, and red for A2440A, A2440B, and $\mathrm{A} 2440 \mathrm{C}$, respectively). The histograms indicate that most galaxies in A are in the velocity bin at $28000 \mathrm{~km} \mathrm{~s}^{-1}$ (EMMIX partition 2). Within the limits of the small number of counts per bin, there is no evidence of another significant segregation in velocity for galaxies in the regions $B$ and $C$ : the velocity distribution seems spatially mixed.

The results of the ROSTAT analysis for the regions corresponding to the three X-ray subclusters are listed in Table 2. Both the mean velocity and velocity dispersions of the $B$ and $C$ regions are comparable, and consistent with the global cluster values. This suggests two possible alternatives: either A2440B and $\mathrm{A} 2440 \mathrm{C}$ have not yet interacted, and the merging is occurring in the plane of the sky, or they have already crossed, but are seen just after core passage. The velocity of the BCG in the $B$ region, $\mathrm{BG} 2$, is close to the velocity of its host subcluster, while BG3 in the $C$ region has a $(\sim 2 \sigma)$ velocity offset of $\left(418 \pm 188 \mathrm{~km} \mathrm{~s}^{-1}\right)$. The velocity dispersions of the two main components A2440B and A2440C are higher than the values expected from their X-ray temperature (see Fig. 6), but the deviation from the $\sigma-T_{\mathrm{X}}$ relation is not statistically significant.

The mean velocity of A2440A is $\approx 750 \mathrm{~km} \mathrm{~s}^{-1}$ higher than that of the main cluster at a $3 \sigma$ level. The small velocity dispersion of $A$ is more typical of a group. The velocity of the brightest galaxy in $A, V G 1=27925 \mathrm{~km} \mathrm{~s}^{-1}$, is consistent with the mean velocity of its subcluster, implying that it is at rest in the potential well. This dynamical analysis is in very good agreement with that obtained by Beers et al. (1991) and Mohr et al. (1996).

These results show that A2440A is a group at higher velocity with respect to the main system, which includes the two subclusters A2440B and A2440C: these two components must have recently crossed each other or are close to merging in the plane of the sky.

We also applied the normality tests to the three subclusters. In the case of the $A$ subcluster, the 10 ROSTAT tests and Dip test exclude normality at more than the $10 \%$ level. This result remains unchanged when excluding a low velocity galaxy that is $3000 \mathrm{~km} \mathrm{~s}^{-1}$ offset from the major velocity peak. We note however that the analysis of this subcluster relies on only 11 (10) velocities. For subclusters $B$ and $C$, all the normality tests (except for one in the case of $B$ ) and the Dip test are consistent with a unimodal Gaussian distribution.

\subsection{Dynamical analysis}

\subsubsection{Mass estimates}

We estimated the mass of the three components in A2440 following the same method as for A2933. However, to determine the harmonic radius we selected the galaxies in the red sequence, defined using our $B$ and $R$ photometric catalogues, with $R<20$; in this way, background contamination was minimized. For each component, we selected galaxies satisfying the above criteria within a radius of 3.0 arcmin from the respective BCG.

As a caveat, we emphasize that we consider subclusters potentially in a post-merger phase, in which case one expects important distortions in morphology and velocity field. However, previous analyses have shown that the velocity distributions in both components B and C can be assumed to be Gaussian. We therefore assume that the two main components, A2440B and A2440C, are now not far from dynamical equilibrium. In contrast, A2440A has a non-Gaussian velocity distribution, but this was determined using only a few redshifts. However, its characteristics are similar to those of a group, and its contribution to the whole mass should be a priori negligible. 
We find for A2440A, A2440B, and A2440C, respectively, that $M_{200}=0.1 \pm 0.02 \times 10^{14} M_{\odot}, M_{200}=5.6 \pm 1.1 \times 10^{14} M_{\odot}$, and $M_{200}=5.4 \pm 1.1 \times 10^{14} M_{\odot}$. As previously discussed, $B$ and $C$ appear to be the main subclusters; the estimated total mass of A2440 is $M_{\mathrm{A} 2440} \sim 1.2 \times 10^{15} M_{\odot}$.

\subsubsection{A2440: two-body model}

The two-body model was applied to the central system composed of $\mathrm{A} 2440 \mathrm{~B}$ and $\mathrm{A} 2440 \mathrm{C}$, with input parameters $R_{\mathrm{p}}=$ $0.442 \mathrm{Mpc}$ and $V_{\mathrm{r}}=200 \mathrm{~km} \mathrm{~s}^{-1}$ derived from the BCGs. As expected in terms of both projected separation and velocities, the two-body model is not well constrained by the observations. Bound solutions exist in both the pre-merger case with $t_{0}=12.2 \mathrm{Gyr}$ (two incoming and one outgoing), and in the postmerger case (one outgoing for $t_{0}<0.5 \mathrm{Gyr}$, two incoming, and one outgoing for $t_{0}>0.5 \mathrm{Gyr}$ ). Therefore, according to the twobody analysis, A2440B and A2440C may be systems seen either before or after first encounter.

We also tested the two-body system composed of A2440A and the whole complex $\mathrm{A} 2440(\mathrm{~B}+\mathrm{C})$. To estimate $R_{\mathrm{p}}$ and $V_{\mathrm{r}}$, we used the position and velocity of the centroid of the whole system A2440 $(\mathrm{B}+\mathrm{C})$, obtaining $R_{\mathrm{p}}=0.6 \mathrm{Mpc}$ and $V_{\mathrm{r}}=730 \mathrm{~km} \mathrm{~s}^{-1}$. In the pre-merger hypothesis $\left(t_{0}=12.2 \mathrm{Gyr}\right)$, there are two bound incoming (BIa, BIb), and one bound outgoing (UO) solutions (Fig. 14). If seen after first passage, one bound outgoing solution exists (BO). The different solutions are listed in Table 4.

\subsection{The scenario for $A 2440$}

We can now use the complete set of information collected from our analysis of A2440 to determine the merging scenario. The bimodal X-ray emissivity of the main component is evidence of the interaction of two massive subclusters being likely to undergo or to have undergone a merging event. At first sight, the existence of two units with cool cores, and a close correspondence between the gas and bright galaxy distributions, which are well centered on the BCGs, are indicative of a pre-merger event. This was also the conclusion of Mohr et al. (1996) for A2440, who observed the similarity between galaxy projected density maps and X-ray emission maps from Einstein.

However the pre-merger hypothesis is strongly disfavored by the lack of any strong temperature enhancement between the two maxima, as we would expect in a pre-merger event (RS01). The similar velocity distributions of the two subclusters can be understood in terms of both a pre-merger and post-merger, but with different implications: in the pre-merger case it is indicative of a merging in the plane of the sky, while in the post-merger case it is due to a very recent collision. The observed difference of clustering for faint and bright galaxies, with a good coincidence between the gas and bright galaxies centroids for A2440-B and A2440-C subclusters, but a segregation between the centroids of the gas and faint galaxies distributions also indicate that merging has already occurred. These results have already been found for several post-merger clusters (Biviano et al. 1996; Maurogordato et al. 2008).

The evidence of a cold front delimiting the core of the Southern subcluster also favors a post-merger scenario, where the stripped subcluster is now moving away from its companion, as shown for instance in A1201 by Owers et al. (2009). The velocity offset of BG2 with respect to A2440-C also implies that there is some dynamical activity in this region. To make this scenario compatible with the observed bimodal structure, we argue that the collision must have occurred with a relatively high impact parameter because a too small impact parameter would have led to the disruption of the two former subcluster cores during the violent relaxation of the gas. The similar values obtained for the temperatures and velocity dispersions of A2440B and A2440C suggests that these components have comparable masses. If one identifies the hot structures surrounding A2440B and A2440C as remnants of a shock wave, a scenario with an equal mass collision with a high impact parameter after the maximum core collapse, when the shock wave begins to propagate towards the outskirts of the new structure, is therefore very likely to explain the $\mathrm{A} 2440(\mathrm{~B}+\mathrm{C})$ complex.

The A2440A subcluster is probably a group, with a significant velocity offset $\left(\sim 750 \mathrm{~km} \mathrm{~s}^{-1}\right)$ with respect to the main cluster A2440 $(B+C)$. The coincidence of the BCG position with the maximum of the gas and galaxy distribution and the consistency of the BCG velocity with the subcluster mean velocity, suggest that the BCG is at the center of the subcluster in dynamical equilibrium. We should expect a Gaussian velocity distribution for this subcluster, which is not the case: this may be due to the small number of available redshifts (10) and contamination from galaxies not belonging to A2440A.

The lack of signatures of gas compression between A2440A and A2440B excludes a pre-merger hypothesis implying small physical separations (BIa). The two viable pre-merger solutions are then incoming (BIb) or outgoing (BO), both with a major component along the line of sight ( $\alpha \sim 80$ degrees). The postmerger case remains unlikely, as there is a good correspondence between the gas and galaxy distributions and the X-ray temperature map does not show any evidence of shocked gas in the periphery of A2440A. Therefore A2440A is probably a group infalling for the first time into the main cluster component along the NE/SW axis.

\section{A2384}

\subsection{X-ray gas morphology and thermal structures}

Looking at Fig. 9, it is clear that the X-ray morphology of A2384 is very peculiar. The general shape of the whole cluster is very elongated, including a northern primary maximum, connected to a southern secondary maximum by a continuous gas distribution. The temperature of this gas is around 3-4 keV, while its surroundings are hotter (up to $4-5 \mathrm{keV}$ ). Both subclusters exhibit cool cores.

\subsection{Galaxy density distribution}

A2384 is one of the best examples of colinear distribution of substructures in a cluster (see West et al. 1995). At all magnitude limits, the galaxy distribution shows a very elongated structure, extending over 1.2 Mpc along the N-S axis (Fig. 1); embedded in this structure, there are two main subclusters roughly centered on two bright galaxies (which we call BCG1 and BCG2 in the following; see Fig. 15). The northern subcluster is more densely populated than the southern one. At bright magnitudes $(R<18)$, the galaxy distribution is strongly concentrated in the northern (A2384N) component. Besides BCG1, in A2384N there are two other very bright galaxies, while in A2384S there are no bright galaxies around BCG2. Near A2384N, a clump of galaxies to the west causes an elongation in the density map. While A2384S is very elongated along the general N/S axis of the system, the internal contours of $\mathrm{A} 2384 \mathrm{~N}$ are oriented towards the NW/SE, and rotate to the $\mathrm{W}-\mathrm{E}$ and general $\mathrm{NE} / \mathrm{SW}$ direction on larger scales. 


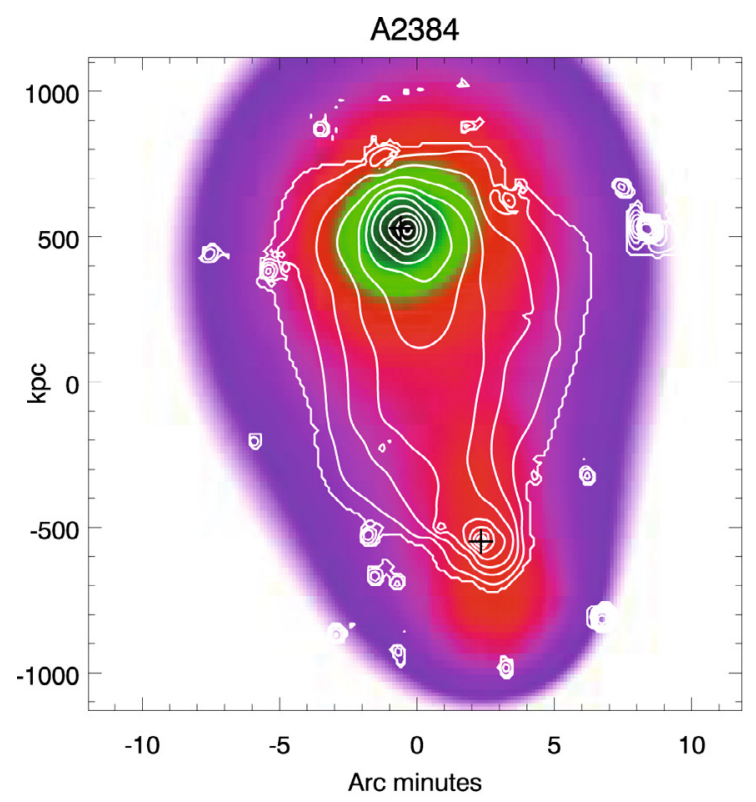

R.A.(0) $215219.73 \operatorname{Dec}(0)-193804.2$

Fig. 9. Same as Fig. 8 for A2384.
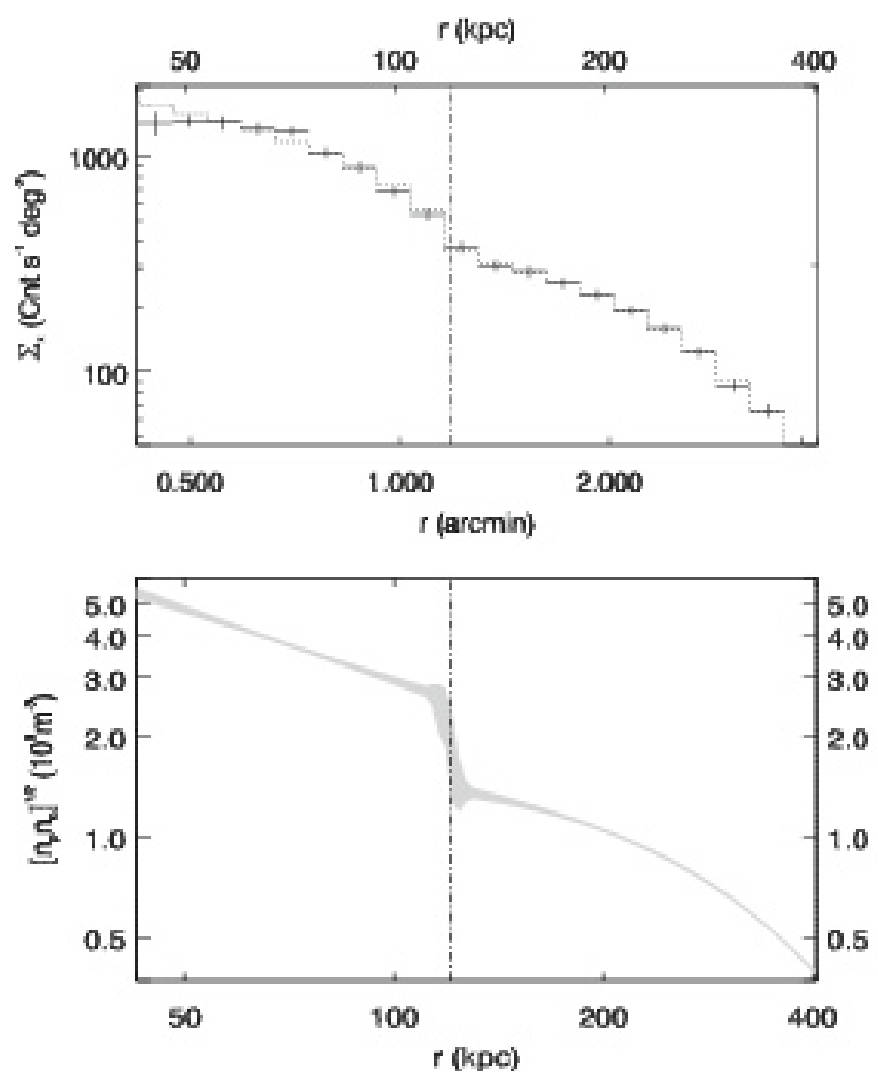

A2384

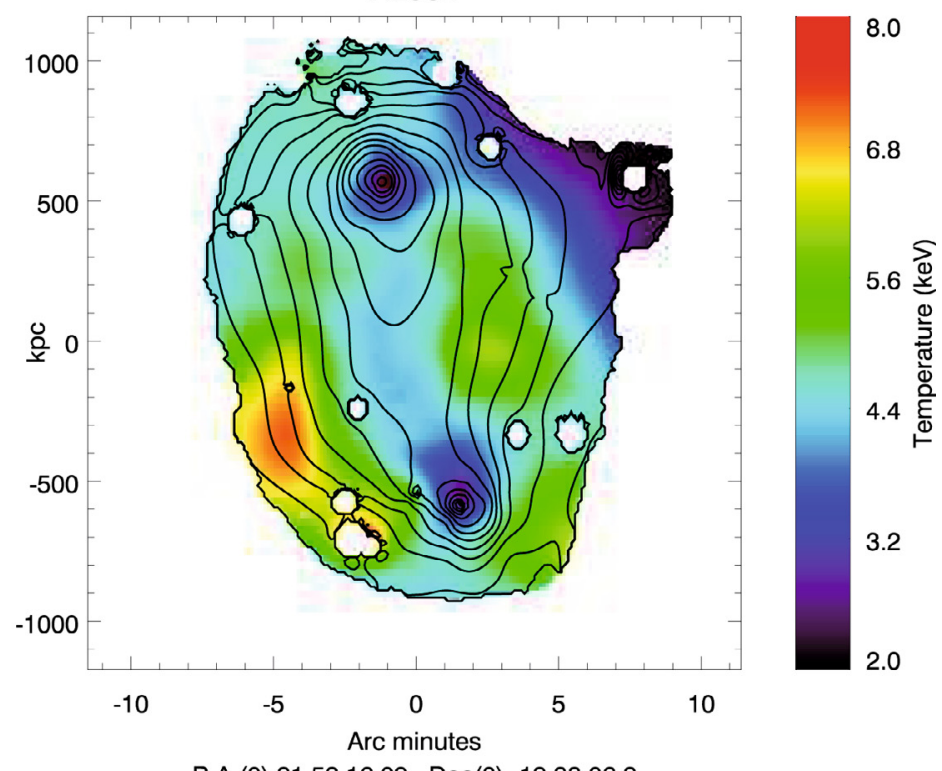

R.A.(0) $215216.09 \operatorname{Dec}(0)-193806.2$
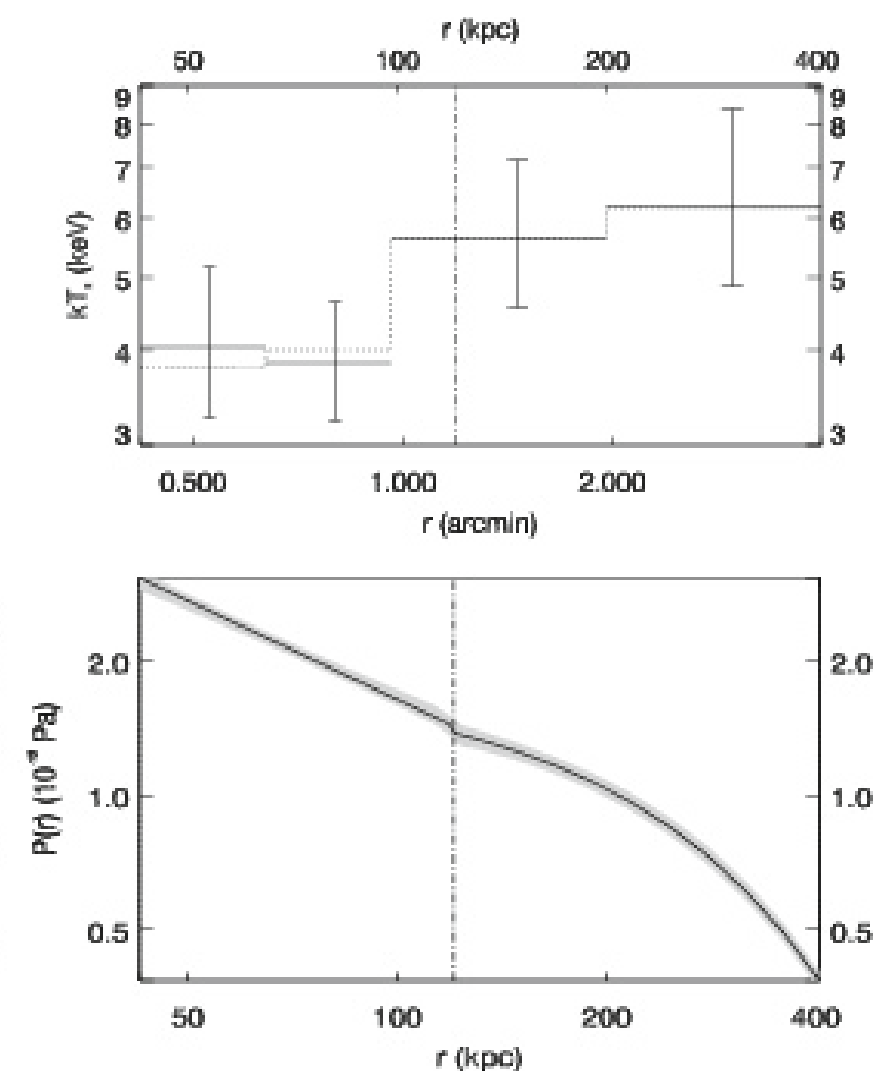

Fig. 10. Top: gas brightness and projected temperature profiles observable across the white sector in Fig. 8 for A2440. Bottom: ICM density and pressure profile estimated above the Southern gas clump in A2440. These profiles reveal a density discontinuity at constant pressure or "cold front" feature (see vertical dashed lines on the plots).

When including fainter objects, the isodensity contours of the two components are elongated towards each other (see Fig. 1).

\subsection{Galaxy velocity distribution}

Before our observations, very few redshifts were available in the literature for this cluster. In our $30^{\prime} \times 30^{\prime}$ field, only 4 galaxies already had a redshift; we reobserved two of them, and our redshifts are in agreement with the literature values within the estimated errors. Our final catalog includes 84 redshifts, and the number of cluster members is 56 .

The mean location of the cluster, $\mathrm{CBI}=28263 \pm 154 \mathrm{~km} \mathrm{~s}^{-1}$, is well defined, but the scale is quite high (SBI $=1114 \pm$ $120 \mathrm{~km} \mathrm{~s}^{-1}$ ). The velocity distribution (Fig. 16) is very broad 


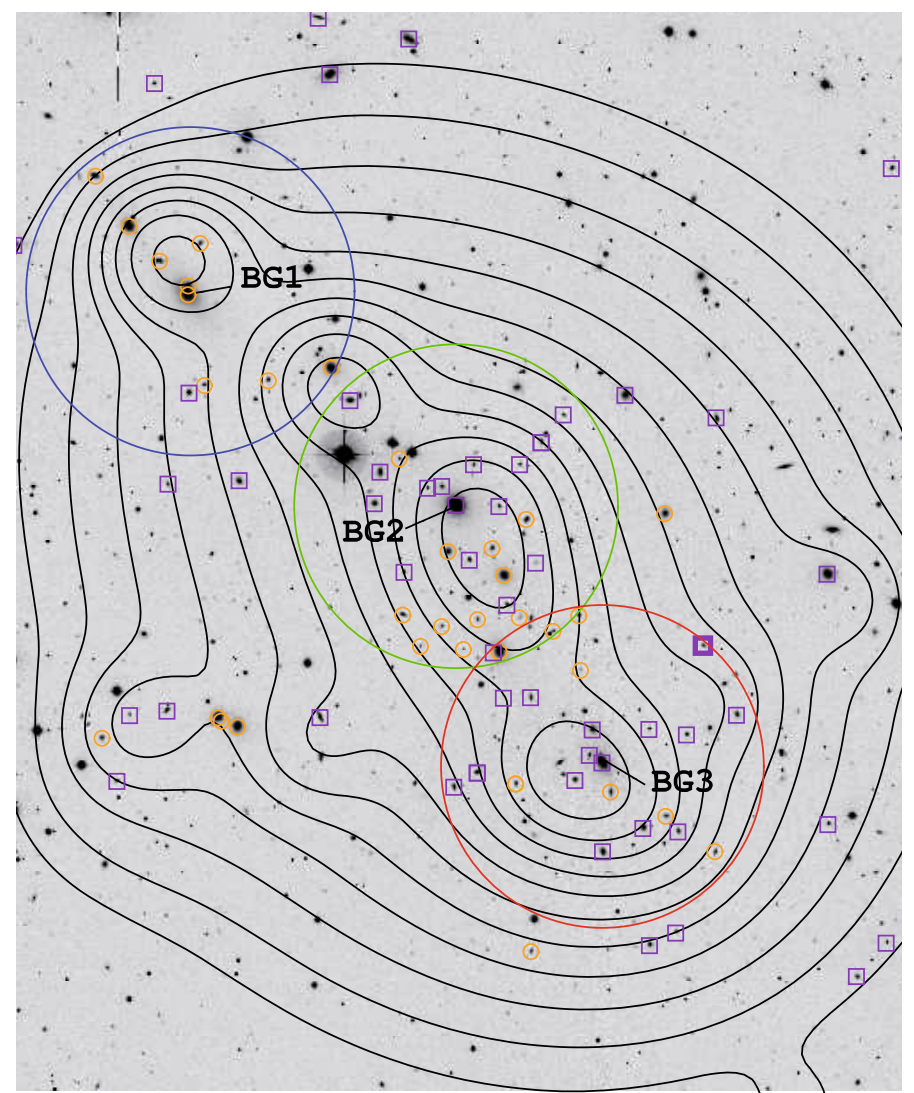

Fig. 11. WFI $R$-band image of the A2440 field $\left(12^{\prime} \times 14^{\prime}\right)$. Galaxies identified as cluster members from spectroscopy (Flag 0 and 1) are marked with different symbols according to the velocity range (purple squares: [24 000, 27600$] \mathrm{km} \mathrm{s}^{-1}$; orange circles [27600, 30000] $\mathrm{km} \mathrm{s}^{-1}$ ). The isocontours of red sequence galaxy density maps with magnitude limit $R<19$ are superimposed. The velocity histograms of the three regions with 2.5 arcmin radius delimited by circles (corresponding to the three subclusters A2440A, A2440B, and A2440C) are displayed in Fig. 13. North is up and East is to the left.

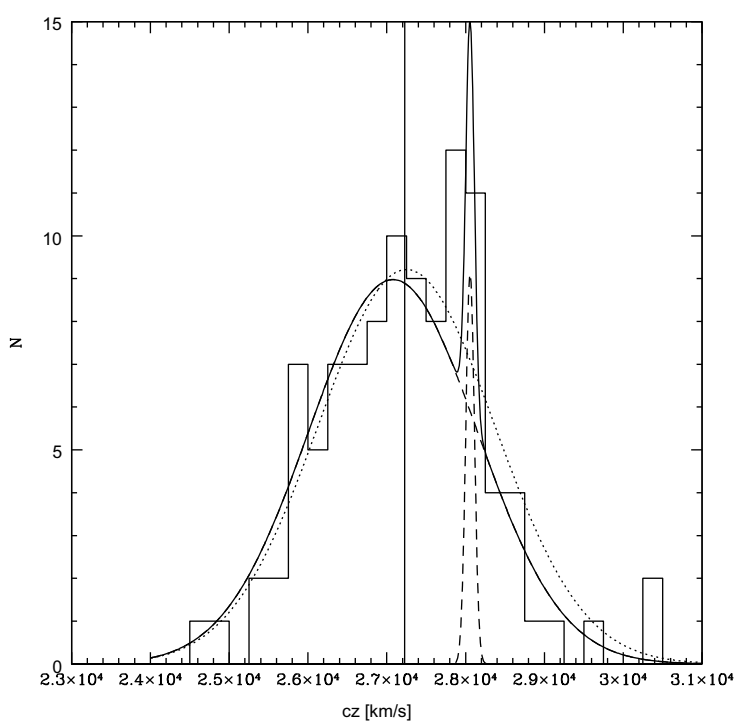

Fig. 12. Velocity histogram of A2440 (binning of $250 \mathrm{~km} \mathrm{~s}^{-1}$ ). The best Gaussian fit for the whole distribution (dotted line) is centered on the vertical solid line which gives the location value. We also show the two Gaussian functions (dashed lines) corresponding to partitions 1 and 2 in the best 3 partition mixture model by EMMIX and the composite function (solid line).

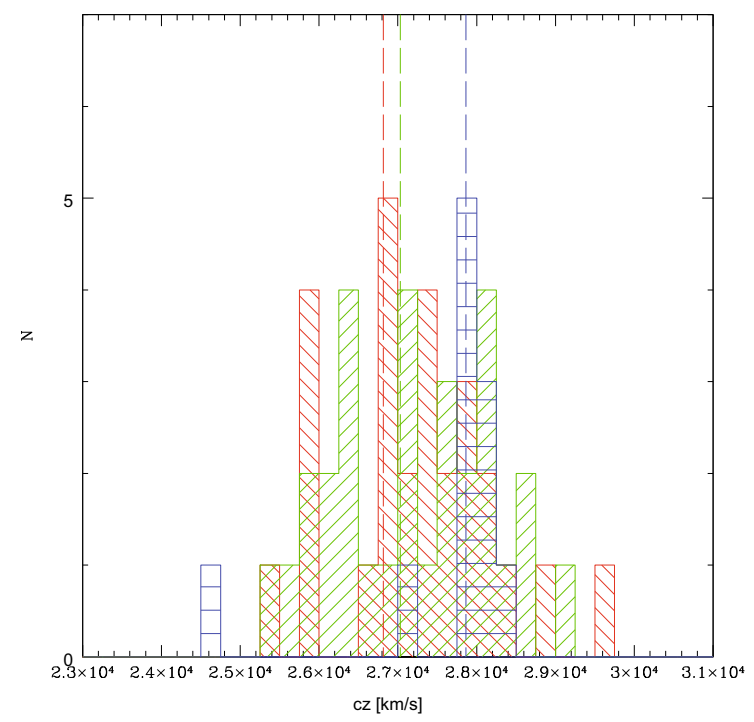

Fig. 13. Velocity histograms of A2440A (blue), A2440B (green) and A2440C (red). For each subcluster we included galaxies within a radius of 2.5' from its center. Dotted lines: velocities of the brightest galaxies in A2440A (blue), A2440B (green) and A2440C (red).

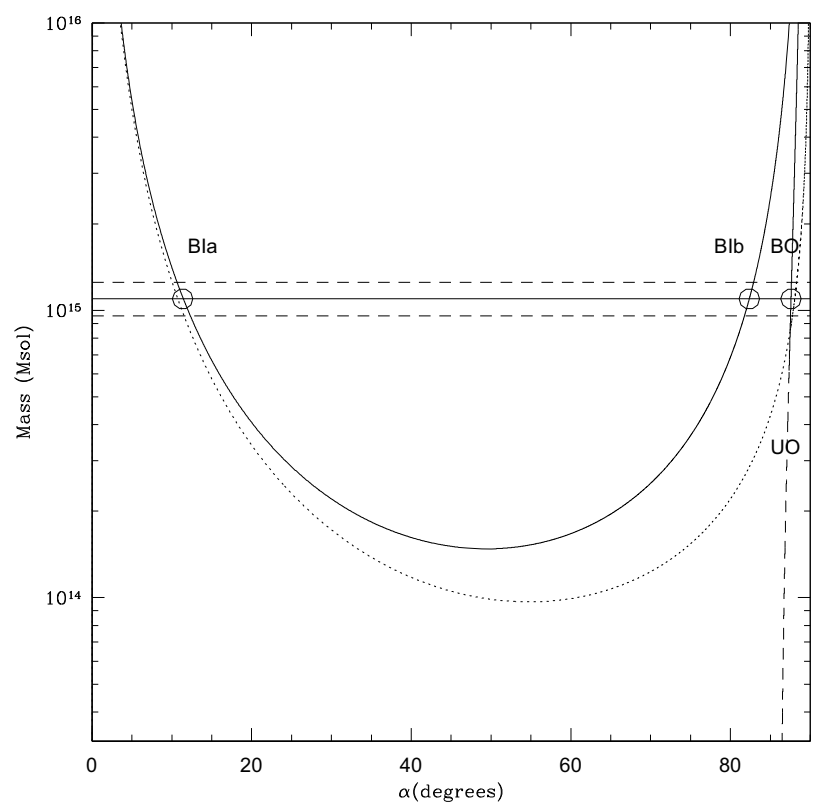

Fig. 14. Two-body model for the system A2440A/A2440(B+C). The horizontal lines show the mass estimate (full line) and errors (dashed lines) (see Sect. 5.5.1). The projected distance of $0.6 \mathrm{Mpc}$ and the radial velocity difference at rest of $730 \mathrm{~km} \mathrm{~s}^{-1}$ were derived from the position and velocity of the two centroids. The two systems are assumed to have been at zero separation $12.2 \mathrm{Gyr}$ ago. The dotted line represents the Newtonian criterion for gravitational binding. Two bound incoming (BIa and BIb) and one unbound outgoing (UO) solutions are found compatible with the mass estimates.

and has three main peaks. This extended velocity distribution could be either due to a very deep potential (massive system) or multi-modality. The hypothesis of a massive system is improbable, given the low cluster optical luminosity and richness. The battery of normality tests do not indicate significant departures from normality. The Dip test excludes unimodality with a $P$-value of 0.1 .

In Fig. 15, cluster members in the three velocity peaks are identified with different symbols and colors. We have very few 


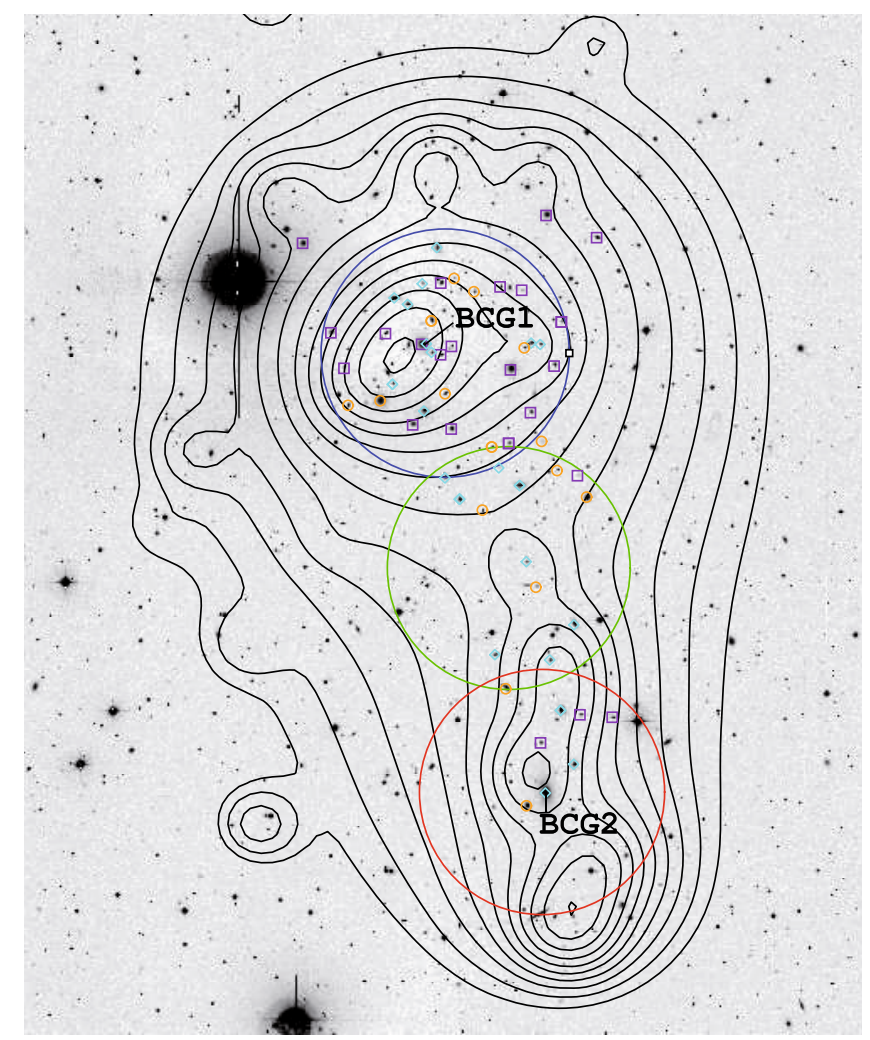

Fig. 15. WFI $R$-band image $\left(20^{\prime} \times 25^{\prime}\right)$ centered on A2384. Galaxies identified as cluster members from spectroscopy (flags 0 and 1$)$ are marked with different symbols as in the previous cases (purple squares: [24 000, 28000$] \mathrm{km} \mathrm{s}^{-1}$, cyan diamonds: [28 000, 29000$] \mathrm{km} \mathrm{s}^{-1}$ and orange circles: $[29000,31000] \mathrm{km} \mathrm{s}^{-1}$ ). Isocontours corresponding to red sequence galaxy density maps at $R<19$ are superimposed. The velocity histograms of the three regions delimited by circles with 3 arcmin radius, which include the two subclusters and the intermediary region, are displayed in Fig. 18. North is up and East is to the left.

redshifts in A2384S, because it is a poor structure. A striking feature is the high fraction of objects in the filament joining $\mathrm{A} 2384 \mathrm{~N}$ and $\mathrm{A} 2384 \mathrm{~S}$, which are associated to the second velocity peak ( 28500 $\left.\mathrm{km} \mathrm{s}^{-1}\right)$.

For A2384, the best-fit solution was found by EMMIX for a partition of three Gaussians roughly centered on the peaks visible in the velocity histogram, with a $P$-value of 0.16 . This indicates that, while the fit quality is greatly enhanced by using 3 Gaussians instead of 1, the null hypothesis of Gaussianity is not rejected. The three Gaussian functions are visualized in Fig. 16; the sum of these functions reproduces the data quite well. The results are summarized in Table 3 . The whole velocity distribution can be reproduced by the combination of two Gaussians with intermediate velocity distributions $\left(\sim 365 \mathrm{~km} \mathrm{~s}^{-1}\right.$ and $\sim 665 \mathrm{~km} \mathrm{~s}^{-1}$ corresponding to partitions 1 and 3 , respectively) at mean velocities $\sim 1200 \mathrm{~km} \mathrm{~s}^{-1}$ higher and lower than the mean velocity of the whole cluster, and by a third strongly peaked component of low dispersion, $\sigma \sim 250 \mathrm{~km} \mathrm{~s}^{-1}$ peaked at $\sim 28500 \mathrm{~km} \mathrm{~s}^{-1}$ (corresponding to partition 2). In the following section, we discuss the spatial distribution of the galaxies assigned to the different velocity partitions with respect to the X-ray and optical density distribution.

\subsection{A2384: X-ray/optical combined analysis}

Looking at Fig. 9, both the gas and the galaxy maps approximately define the same bimodal structure. The northern and southern overdensities (A2384N and A2384S) correspond to the cool cores in the X-ray temperature map. The X-ray maximum of $\mathrm{A} 2384 \mathrm{~S}$ is coincident with the BCG2 position, while BCG1 is slightly offset westwards (15 arcsec) with respect to the X-ray maximum of A2384N (see Fig. 9). This offset cannot be due to astrometric errors, as the precision of the optical images is superior to $1^{\prime \prime}$, while the precision of X-ray images is superior to $4^{\prime \prime}$, and there is a very good correspondence between the optical and X-ray positions of point sources. As BCG1 also has a significant velocity offset with respect to A2384N (see below), this indicates that it is not at rest in the subcluster potential. However, the velocity distribution of $\mathrm{A} 2384 \mathrm{~N}$ has a large dispersion, and at the cluster redshift 15 arcsec corresponds to a physical projected separation of about $25 \mathrm{kpc}$, which means that BCG1 is close to the cluster center, which is probably included in its extended halo.

As already noted, the velocity dispersion of A2384 is unexpectedly high with respect to its optical luminosity and to the low values of its X-ray luminosity and temperature. A cluster with velocity dispersion $\sim 1000 \mathrm{~km} \mathrm{~s}^{-1}$ should have an X-ray temperature $T \sim 7 \mathrm{keV}$, according to the $\sigma-T_{\mathrm{X}}$ relation (Fig. 6), which is much higher than the observed value. Moreover, from a weak lensing analysis Cypriano et al. (2004) fit a velocity dispersion of $737 \pm 126 \mathrm{~km} \mathrm{~s}^{-1}$ or $797 \pm 108 \mathrm{~km} \mathrm{~s}^{-1}$ depending on the assumed profile (SIS and SIE, respectively). These velocity dispersions corresponds to $T \sim 3.45 \mathrm{keV}$ and $T \sim 4.02 \mathrm{keV}$, respectively, which are consistent with the observed values in X-rays. This suggests that the measured velocity dispersion is overestimated.

We analyzed the velocity distribution in A2384N and A2384S, selecting galaxies in circles of radius 5 arcmin centered on the X-ray maxima. The velocity histograms in the corresponding regions are shown in Fig. 17. The mean velocity of $\mathrm{A} 2384 \mathrm{~S}$ is $\sim 675 \mathrm{~km} \mathrm{~s}^{-1}$ higher than that of $\mathrm{A} 2384 \mathrm{~N}$. The same trend is followed by the BCGs of the two subclusters, which 
S. Maurogordato et al.: Merging history of three bimodal clusters

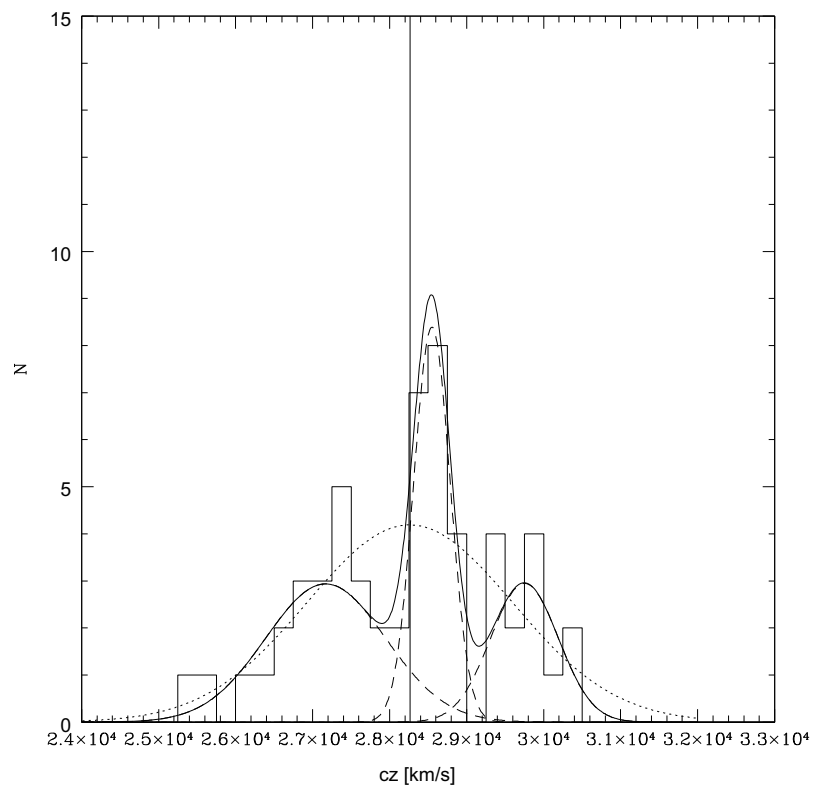

Fig. 16. Velocity histogram of A2384 (binning of $250 \mathrm{~km} \mathrm{~s}^{-1}$ ). The best Gaussian fit for the whole distribution (dotted line) is centered on the vertical solid line which gives the location value. Location and scale of the Gaussian were estimated with ROSTAT. We also show the three Gaussian functions (dashed lines) corresponding to the best mixture model by EMMIX and the composite function (solid line).

show an even larger offset of $\sim 1000 \mathrm{~km} \mathrm{~s}^{-1}$. We also found that the position of the BCG1 has a small offset with respect to the centroid of $\mathrm{A} 2384 \mathrm{~N}$, while the position of $\mathrm{BCG} 2$ corresponds to the centroid of A2384S. The velocity of BCG1 is $600 \mathrm{~km} \mathrm{~s}^{-1}$ lower than the mean velocity of galaxies in A2384N, while the velocity of BCG2 is consistent with that of A2384S (see Table 2).

The velocity dispersions of the two subclusters are also quite large: $\sim 1200 \mathrm{~km} \mathrm{~s}^{-1}$ for A2384N and $\sim 900 \mathrm{~km} \mathrm{~s}^{-1}$ for A2384S. Both subclusters are above the $\sigma-T_{\mathrm{X}}$ relation; the deviation is at the $3 \sigma$ level for $\mathrm{A} 2384 \mathrm{~N}$, but within the errors in the case of A2384S. ROSTAT normality tests reject the Gaussian hypothesis for neither A2384-N, nor A2384S. The Dip-test however excludes unimodality for A2384-N (A2384-S) with a $P$-value of $0.1(0.01)$. This suggests that the subclusters are still dynamically perturbed.

We also defined three subsamples by selecting galaxies within a radius of 3 arcmin from the centers of A2384N, A2384S, and the intermediate region (see Fig. 18). The galaxies in the northern component show a large spread in their velocity distribution, which is skewed towards lower values and has a peak at $\sim 28500 \mathrm{~km} \mathrm{~s}^{-1}$, while galaxies in the southern and especially in the intermediate region are more strongly concentrated in the same peak; this peak corresponds to EMMIX partition 2.

\subsection{Dynamical analysis}

\subsubsection{Mass estimates}

We applied a method similar to that applied to A2440, determining the harmonic radius of each component by selecting only the galaxies in the red sequence, with $R<20$ and within a radius of 5 arcmin from the respective BCG.

However, as we previously discussed, the velocity distribution of this system is not simple. Our mass estimates could therefore be severely affected. We find for A2384N and A2384S that

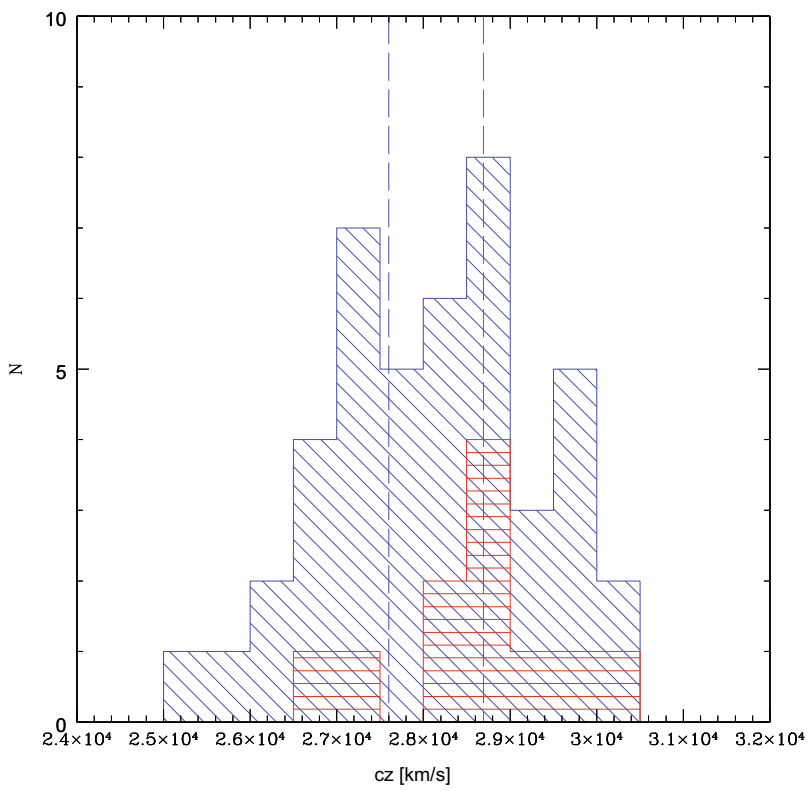

Fig. 17. Velocity histograms of A2384N (blue), and A2384S (red). For each subcluster we included galaxies within a radius of $5^{\prime}$ from its center. Velocity bins of $500 \mathrm{~km} \mathrm{~s}^{-1}$ are used. Dashed lines: velocities of the brightest galaxies in A2384N (blue) and A2384S (red).

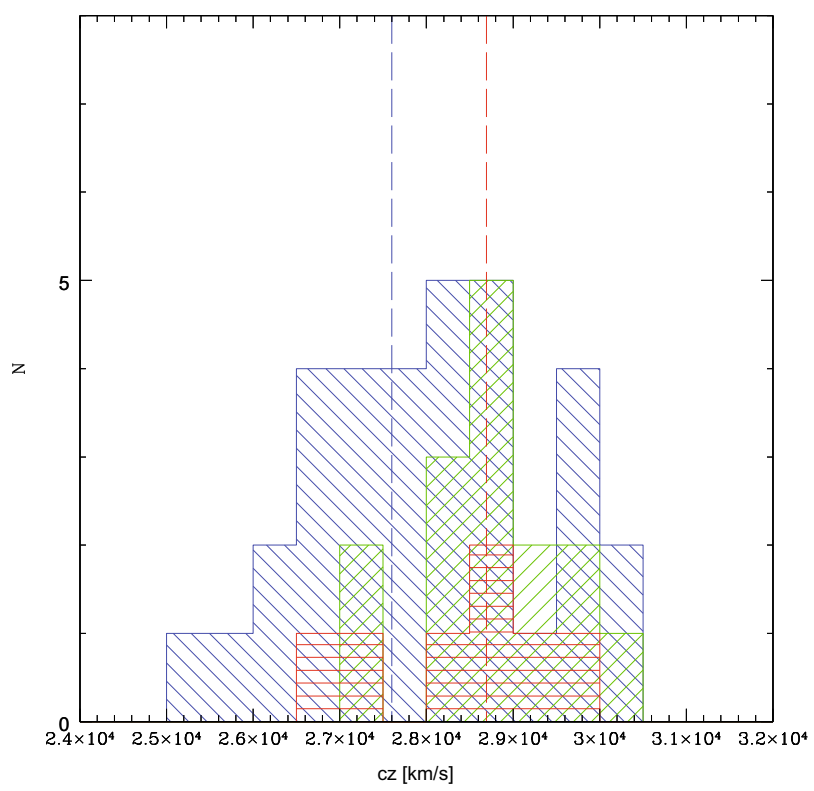

Fig. 18. Velocity histograms of A2384N (blue), the intermediate region (green) and A2384S (red). For each subcluster we included galaxies within a radius of $3^{\prime}$ from its center. Velocity bins of $500 \mathrm{~km} \mathrm{~s}^{-1}$ are used. Dashed lines: velocities of the brightest galaxies in A2384N (blue) and A2384S (red).

$M_{200}=1.4 \pm 0.3 \times 10^{15} M_{\odot}$ and $M_{200}=0.6 \pm 0.1 \times 10^{15} M_{\odot}$, respectively. As the velocity dispersion is probably overestimated, we consider in the following $M_{200}=2.0 \times 10^{15} M_{\odot}$ as an upper bound to the sum of the mass of the two components.

\subsubsection{Two-body model}

We applied the two-body model to A2384N and A2384S, assuming as values of $R_{\mathrm{p}}$ and $V_{\mathrm{r}}$ the projected separation and relative velocity at rest of the BCGs $\left(R_{\mathrm{p}}=1.14 \mathrm{Mpc}, V_{\mathrm{r}}=1000 \mathrm{~km} \mathrm{~s}^{-1}\right)$. We tested several scenarios: the pre-merger case $\left(t_{0}=12.2 \mathrm{Gyr}\right)$, 


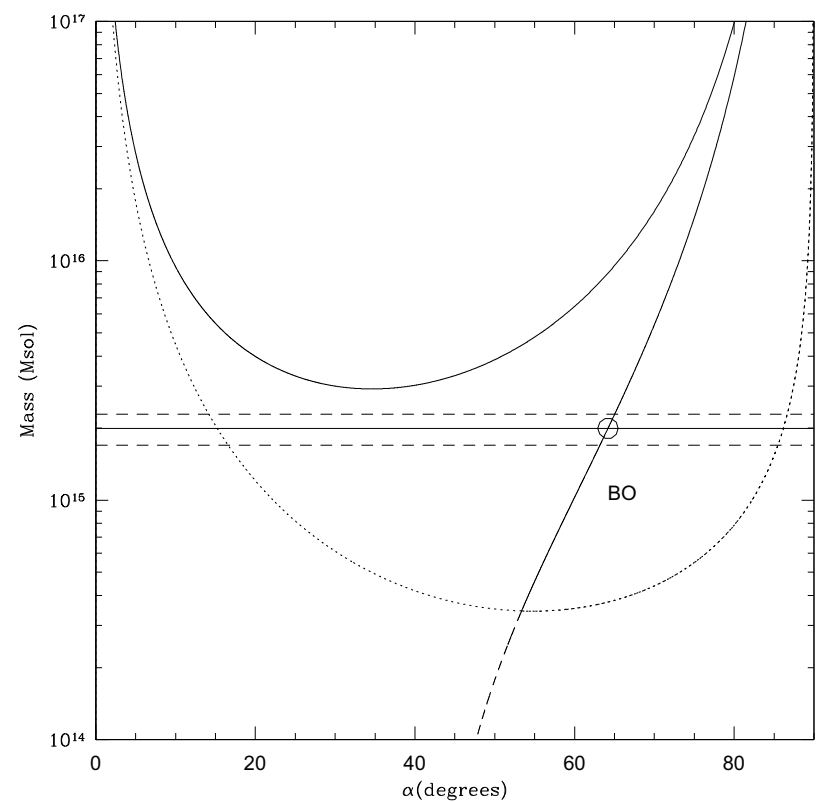

Fig. 19. The sum of the virial masses of $A 2384 \mathrm{~N}$ and A2384S as a function of the projection angle $\alpha$. The horizontal lines show the mass value (full line) and errors (dashed lines) as estimated in Sect. 6.4. The projected distance of $1.14 \mathrm{Mpc}$ and the radial velocity difference at rest of $1000 \mathrm{~km} \mathrm{~s}^{-1}$ were derived from the two BCGs positions and velocities. It is assumed that the two systems are $1.0 \mathrm{Gyr}$ after the first passage. The dotted line represents the Newtonian criterion for gravitational binding. One unbound outgoing (UO) is found compatible with our mass estimates.

and a range of post-mergers $\left(t_{0}=0.2,0.5,1 ., 1.5\right.$ and $\left.2 \mathrm{Gyr}\right)$. In the pre-merger case, two incoming bound solution and one unbound solutions were found. In the post-merger case, only an unbound solution exists if the merging is recent $\left(t_{0}<0.5 \mathrm{Gyr}\right)$, and a bound outgoing solution exists if the merger is older $\left(t_{0}>0.5 \mathrm{Gyr}\right)$. Figure 19 shows the case of a post-merger seen $1.0 \mathrm{Gyr}$ after the encounter for which a unique bound outgoing solution exists. At $t_{0}>1.5 \mathrm{Gyr}$, two bound incoming solutions and a bound outgoing solution are possible.

\subsubsection{A2384 proposed scenario}

The bimodal structure of A2384 detected in both the galaxy and gas density maps suggests that the two components either are about to or have undergone a merger event. The presence of two well-separated components with cold cores, coincident in the X-ray and the optical, is often observed in the case of pre-mergers.

However, the trail of gas and the bridge of galaxies extending from A2384N to A2384S are difficult to explain if the subclusters have not yet interacted. If A2384 were a pre-merger, its temperature map should exhibit a compression region between the two subclusters, which is not the case. The velocity distribution shows a large dispersion $\left(\sigma \sim 1200 \mathrm{~km} \mathrm{~s}^{-1}\right)$, inconsistent with the value of the temperature if it simply reflects the mass of the system. This high value of the velocity dispersion and the velocity distributions of the two components being mixed imply that the merging has already occurred. A2384N shows several signatures of previous dynamical activity: a slight segregation between gas and galaxies, an offset of the position of the BCG from to the peak of the X-ray emitting gas, and of its velocity from the mean velocity of the subcluster. This suggests that the dynamical state of $\mathrm{A} 2384 \mathrm{~N}$ is still disturbed possibly due to a previous collision with A2384S or/and a secondary merger event within A2384N itself.

At first sight, A2384S is more regular than A2384N. It has a good spatial coincidence between its gas and galaxy distributions, coincidence between the position and velocity of the BCG and the X-ray center and mean velocity of the subcluster, respectively. Its velocity distribution is very broad and non-Gaussian, but this may be due to contamination by objects in the periphery of A2384N.

This complex structure could be understood in a scenario in which a low mass cluster of galaxies has crossed the environment of a more massive one and has been stripped of a large fraction of its gas and galaxies. The "head" of the system would then correspond to A2384S, and the tail of galaxies belonging to the original cluster affected by $\mathrm{A} 2384 \mathrm{~N}$ would lie in the intermediary region.

We conclude that the most likely scenario for A2384 is a post-merger between two unequal mass clusters. However, if the merging has already occurred, we have to explain why the two cool cores have remained unaffected by the merging process. Numerical simulations (Poole et al. 2006) have shown that the initial cool cores of the primary and secondary components survive the first core crossing, and disappear after the second pericentric passage.

It is however difficult to constrain the parameters of the scenario, as the mass of A2384 is not well determined. From the 2-body analysis, bound solutions exists only if the merger event is older than 0.5 Gyr. Older mergers $(1-1.5 \mathrm{Gyr})$ with larger physical separations between the units are favoured by the lack of signatures of compression in the X-ray gas (Table 4). For mergers older than $1.5 \mathrm{Gyr}$, one can see that incoming solutions, corresponding to second infall, are again possible. However, these also predict small values of the real separation between the two components, which are unlikely from the lack of signature of compression of the gas in the temperature maps, as noted before. One is then left with the most probable hypothesis of two bound outgoing subclusters seen more than 1.0 Gyr after the first passage, with a collision near the line of sight. Figure 17 shows as an example the bound outgoing solution in a $1.0 \mathrm{Gyr}$ post-merger, which is in good agreement with both our X-ray and optical observations.

\section{7. "Idealized" simulation and XMM emulation}

In the previous sections, we combined optical and X-ray observations to constrain the merging scenario for each one of our three bimodal systems. To help define the scenario and test its consistency, we tried to reproduce the observed X-ray properties with numerical simulations. Adopting an approach similar to RS01, we placed two clusters in a box and allowed them to evolve under gravity in an adiabatic framework. The initial parameters of the collision are inferred from the X-ray and optical observational constraints.

To this aim, we developed a set of programs that allow us to reproduce the observational process and analyze the simulated clusters in the same way as we analyze real ones, adopting an approach similar to e.g. Gardini et al. (2004) for XMM observations.

Using an emission plasma model, where gas pressure and density are known everywhere, we can compute the emitted $\mathrm{X}$-ray photons as a function of telescope area and exposure time. Fixing the direction of observation, we collect photons from the different cells along the line of sight, taking into account that we 
observe clusters in projection (as long as we can assume that the thin plasma approach is valid).

The first step in developing a good "idealized" merger simulation is to check the way we implement the hydrostatic equilibrium in each (sub)cluster (hereafter "unit") to be sure that, during collision, what we see is really due to the collision and not a spurious result of numerical errors in the initial units. Once this is achieved (by allowing a unit to evolve alone in the middle of the box for a very long time), we are able to build collision simulations.

The total mass profile of our "perfect cluster" follows a classic NFW profile, $\rho_{\mathrm{dm}}(r) \propto \frac{1}{r\left(1+r / r_{\mathrm{S}}\right)^{2}}$ (Navarro et al. 1996), while the gas follows a profile described in Suto et al. (1998) adapted to keep the gas mass fraction constant at large radii. The dark matter profile is derived from the difference between the total mass profile and the gas. We note that the NFW profile is derived from pure dark matter simulations, thus this approach is likely to be safe as long as the gas mass fraction is not too high (thus not in the central region of cooling-flow clusters). The velocity dispersion of dark matter particles is computed (as in RS01) using the virial equation $\frac{\mathrm{d}}{\mathrm{d} r}\left[\rho_{\mathrm{dm}}(r) \sigma^{2}(r)\right]=-\frac{G M(r)}{r^{2}} \rho_{\mathrm{dm}}(r)$.

After fixing the gas-to-mass ratio, the concentration parameter $c_{200}=\frac{M_{200}}{r_{\mathrm{S}}}$, the mass ratio between units and the impact parameter (defined as the minimal distance between the two initial trajectories in unit of the NFW scaling radius $r_{\mathrm{S}}$ ), we reconstruct a pre-collision scenario by computing two units as described above. We place these two units in a rather large box to avoid any border effects. We compute the initial velocity considering the pre-merger evolution as a free fall of the two units, exactly in the way described in RS01. We then compute the evolution of the system with the AMR hydro-NBody simulation RAMSES (Teyssier et al. 2002). At each time step, for all the cells in the simulated volume, we store the pressure, velocity, and density of the gas, and position and velocity of dark matter particles. After running these simulations, the following steps are the computation of the photons emitted by the simulated volume and their ray-tracing using the EPIC/XMM instrumental response. These steps are fully described in Bourdin et al. (2004). We finally have a dataset that can be reduced in the same way as real XMM/EPIC observations.

All these previously described steps are important for a correct comparison between observation and simulation. Because of the complexity of this procedure, it is impossible to explore the whole parameter space when determining the optimal collision parameters. By looking simultaneously at the brightness, temperature maps of the X-ray gas, and galaxy density maps, we first guess a set of possible parameters that we place into the complete simulation stream and iterate manually to optimize the set of parameters. Therefore, we do not pretend to fit the data, but we attempt to obtain a scenario reproducing the main features of the observed merging system.

\section{Comparison with simulations and discussion}

Taking as input the scenario inferred from observations for the main merger event, we obtained density and temperature maps as they would be observed with XMM, and compared them with the real observations.

In the case of A2933, the most likely scenario is a pre-merger of two components of comparable mass for which there is a small angle between the collision axis and the plane of the sky. We simulated an equal-mass merger in the plane of the sky with an impact parameter set to zero, and allowed it to evolve with

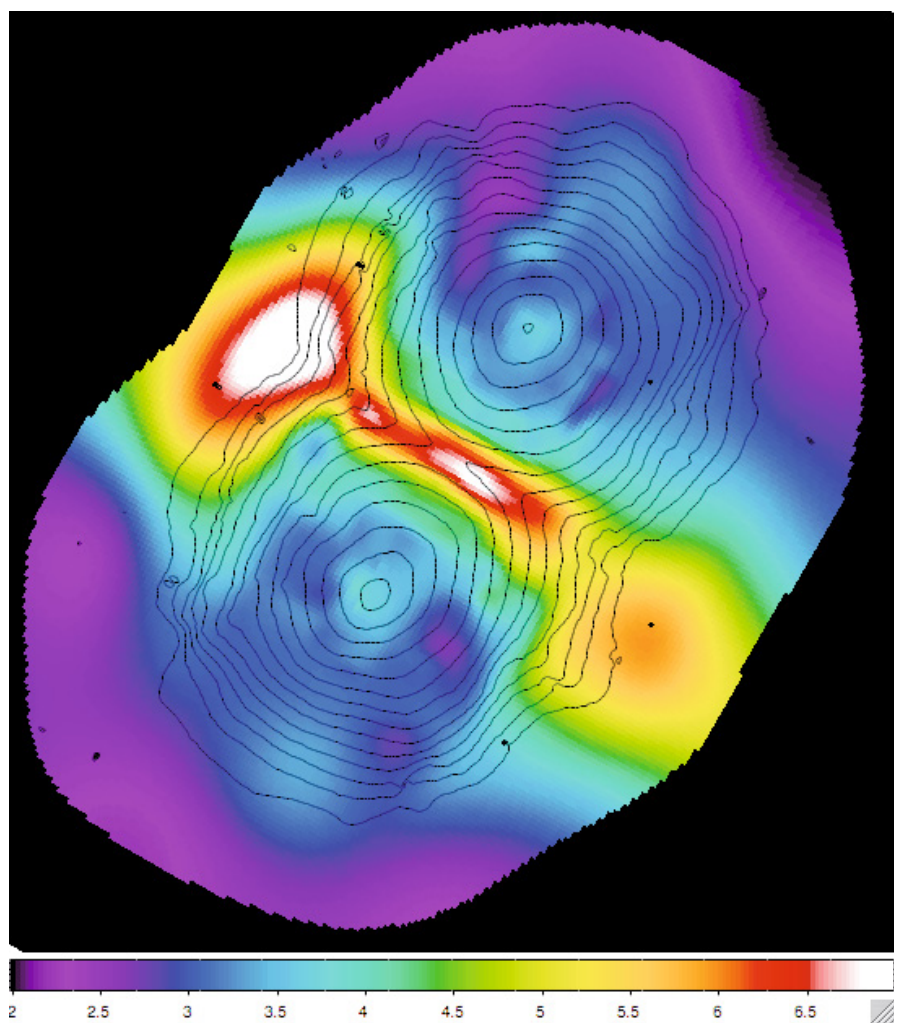

Fig. 20. Simulated wavelet reconstructed temperature map with luminosity contours superimposed, derived from the simulation which best reproduces the observed features of A2933.

time. The resulting temperature map and luminosity contours for this scenario are presented in Fig. 20. The X-ray properties, in particular the temperature contrast between the high temperature region and the mean temperature of each unit and its relative angular distance, are closely reproduced by a pre-merger scenario $\sim 200$ Myr before the core collapse.

For A2440, which is expected to be a post-merger case, we simulated a variety of equal mass mergers with different impact parameters and tried to find the best configuration reproducing simultaneously the observed temperature and luminosity distributions. In Fig. 21, we present our best candidate. This scenario corresponds to a collision with an impact parameter of $15 r_{\mathrm{s}}$ seen $450 \mathrm{Myr}$ after the maximum core collapse. We managed to reproduce the most striking features revealed by the observations, in particular the hot region surrounding the X-ray maximum of each unit, and the temperature contrast between the hottest region and the colder ones. Since our code follows a purely adiabatic approach, we do not expect of course to reconstruct the two cool cores.

In the case of A2384, we performed different trials allowing the impact parameter value to vary. If the impact parameter is not zero, it should be large enough to allow the gas of the smaller unit to follow the influence of its original potential well after the collision (we note that this is also necessary to explain the remaining "cool" core of each unit). At the same time, the impact parameter should be small enough to enable the gas to be stripped efficiently. In these limits, the gas is stripped along a rather significant distance as observed. Simulations by Poole et al. (2006) show a gas surface density similar to that of A2384, in the case of a 3:1 mass ratio seen at the first apocentric passage of the secondary cluster, at $\sim 1.5$ Gyr after the closest approach (see their Fig. 4 central panel). In these scenarios, it is 


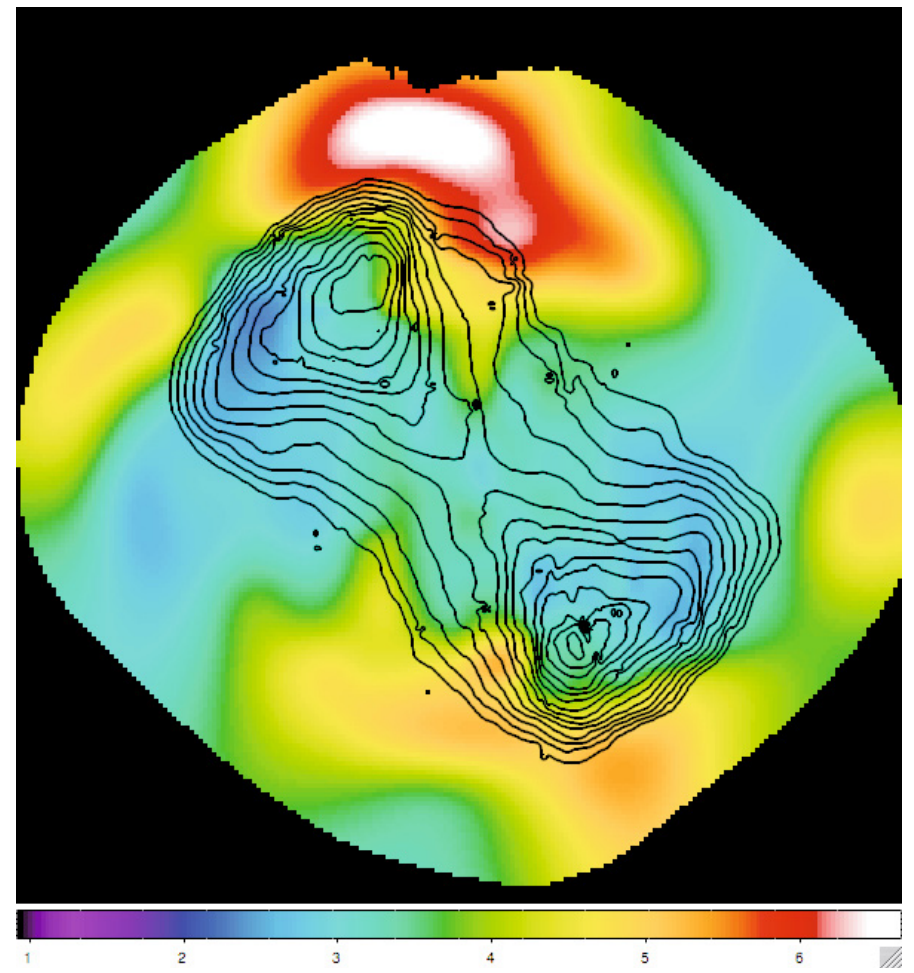

Fig. 21. Simulated wavelet reconstructed temperature map with luminosity contours superimposed, derived from the simulation which best reproduces the observed features of A2440.

nevertheless very difficult to keep the axial symmetry observed in luminosity and in temperature maps and impossible to explain that the BCG of the south unit still lies at the X-ray maximum.

On the other hand, in a null impact parameter collision, if a small group encounters a heavier unit, its gas can escape significantly after the first maximum core collapse only if its central density is very high compare to the central density of the massive object. Simulations by Poole et al. (2006) are able to strip gas even with a null parameter thanks to their initial gas profile $\left(S \propto r^{1.1}\right)$ profile, which leads to a high inner gas density in the core. In contrast, if the small unit has a concentration parameter higher than the massive one (say by a factor 2 ) then the gas appears sticked to its own unit even after the first maximum core collapse. Unfortunately, we cannot simulate such a complex scenario in the adiabatic scheme since we have no cooling thus the inner temperature of the small unit increases strongly with the concentration parameter, preventing the correct description of the gas luminosity and temperature during the collision.

\section{Conclusion}

We have performed a combined X-ray/optical analysis of three bimodal clusters at low redshift, selected as merger candidates from the sample of Kolokotronis et al. (2001). We have confirmed the disturbed dynamical state of these clusters and reconstructed the general trends of their main merging scenario. A2933 has been shown to most likely be an equal-mass advanced pre-merger ( $200 \mathrm{Myr}$ before the core collapse). A2440 is a recent equal-mass merger ( $\sim 50 \mathrm{Myr}$ after core collapse) with another subcluster infalling along the main axis of the cluster, and A2384 is the result of an older collision between two units of mass ratio 1:3 ( 1.0-1.5 Gyr after core collapse).
We emphasize the complementarity of the X-ray and optical data in determining the parameters of the scenario (angle, massratios, epoch) that were refined by running simulations of idealized cluster collisions. Our analysis confirms the efficiency of the selection based on the comparison of gas and density maps to identify merging clusters when (as is frequently the case) information on temperature and velocity distribution is not available. Moreover, the level of segregation between galaxies and gas provides some indication of the merging stage. This approach is far more powerful than the density maps taken individually in either X-rays or the optical. From X-ray density maps only, the three bimodal clusters presented in this paper would have been expected to be in the very last phase before the first maximum core collapse, while two of them are shown to be post-mergers with surviving cores in the two units. The properties of the temperature maps are essential to assess the merging stage: the hot region in between the two sub-clusters in A2933 is indicative of a pre-merger, the spectacular hot structures in the periphery of A2440 and A2384 have been identified as remnants of a postmerger shock wave, and the cold front detected in the S/W region of A2440 is consistent with a a post-merger. On the other hand, redshift information is needed to understand the dynamics of the system, determine the mass of the subclusters and the relative motion of the BCGs, and finally date the merger event and constrain its axis using the two-body analysis.

Numerical simulations have allowed us to refine the collision parameters. Thanks to our XMM simulation tools, we correctly handle all projection and instrumental effects. Thus we can rely on these "observed simulations" and select the most likely scenario based on measured criteria such as distances, temperature, and luminosity. We have been able to reproduce the main features of A2933 and A2440, simulating the collision of two systems initially in complete hydrostatic equilibrium (with an approach similar to RS01) and adding a complete set of programs to reproduce X-ray observations for simulations and obtain EPIC/XMM synthetic observations (with an approach similar to Gardini et al. 2004). The case of A2384 is too complex to be described with our approach, but we have been able to place constraints on its collision parameters.

The results obtained from the analysis of these clusters can be combined to our previous work on other MUSIC clusters to draw a more general picture of merging clusters properties. In the three clusters studied in the present paper, we have shown the existence of preferential axes following the general position angle of the cluster, as previously shown in other merging clusters (Arnaud et al. 2000; Plionis et al. 2003; Maurogordato et al. 2006). Subclusters generally host one or more BCGs. In most cases, the different merging subclusters and their brightest BCGs are aligned along this direction. This corroborates the property of preferential alignment of BCGs with their host cluster (Niederste-Ostholt et al. 2010) on the scale of subclusters. In A2384, we have detected a spectacular filament of galaxies and gas probably stripped from the colliding group along the merging axis.

Another interesting aspect is the position and motion of the BCGs relative to their subclusters. We have found that their angular coordinates generally correspond (within the errors) to the X-ray centroids of their host subclusters (A2933S, A2440A, A2440B, A2440C, A2384S) but in some cases there is a small offset (A2933N, A2384N). Similarly, in some cases the radial velocities of the BCGs are consistent with the mean velocity of their respective subcluster (A2440A, A2440B, A2384S), but in other cases they are offset by $300-500 \mathrm{~km} \mathrm{~s}^{-1}$ (A2933N, A2933S, A2440C, A2384N). These offsets are signatures of 
ongoing dynamical activity in the subclusters, as found in A3921B (Ferrari et al. 2005) and in A2163A (Maurogordato et al. 2008), which were both identified as recent mergers. In an analogous way, the beginning of interaction between A2933N and A2933S, the probable recent mergers occuring within $\mathrm{A} 2933 \mathrm{~N}$ and $\mathrm{A} 2384 \mathrm{~N}$, and the recent merger between A2440B and A2440C can explain the observed BCG offsets.

We have also found that, in addition to the main merging event, a large fraction of MUSIC clusters appear to contain secondary merging events (A2933N and A2384N) and infalling groups, e.g., A2440 (this work), A521 (Ferrari et al. 2003), and A2163 (Maurogordato et al. 2008).

These results are consistent with a hierarchical scenario of structure formation where clusters form by successive mergers and accretion of matter along large-scale filaments, which may cause alignments with structures on various scales (Basilakos et al. 2006; Lee \& Evrard 2007; Faltenbacher et al. 2008).

Moreover, we have also shown that in recent post-merger clusters, such as A2440 (this work) and A2163 (Maurogordato et al. 2008), galaxies exhibit a strong luminosity segregation, similar to the case of the Coma cluster studied by Biviano et al. (1996). We have also found a deviation from the $\sigma-T_{\mathrm{X}}$ relation for the subclusters in a post-merger stage, the largest one (at the $3 \sigma$ level) being detected for A2384N. At variance, both pre-merging subclusters A2933N and A2933S follow the $\sigma-T_{\mathrm{X}}$ relation.

The impact of the merging process on galaxy properties in these clusters, in particular on star formation, will be addressed in a forthcoming paper.

To test the validity of these properties on a large sample, we are currently extending this work to a subsample of the C4 SDSS cluster sample (Miller et al. 2004) with both optical and X-ray available data.

Acknowledgements. The authors want to thank Romain Teyssier for the use of RAMSES Hydro-NBody code and Albert Bijaoui and Eric Slezak for providing their program estimating density maps through a multi-scale approach, and Monique Arnaud for fruitful discussions. We thank the Programme National de Cosmologie et Galaxies of CNRS for his constant support on this program, the Observatoire de la Côte d'Azur and the Laboratoire Cassiopée, CNRS, for specific funding of this project. H.B. acknowledge financial support from contract ASI-INAF I/088/06/0. We also want to thanks an anonymous referee for his/her comments and suggestions which helped us to improve the quality of the paper.

\section{References}

Arnaud, M., Maurogordato, S., Slezak, E., \& Rho, J. 2000, A\&A 355, 461 Barrena, R., Biviano, A., Ramella, M., Falco, E., \& Seitz, S. 2002, A\&A, 386, 816

Barrena, R., Boschin, W., Girardi, M., \& Spolaor, M. 2007, A\&A, 469, 861 Basilakos, S., Plionis, M., Yepes, G., et al. 2006, MNRAS, 365, 2, 539

Beers, T. C., Geller, M. J., \& Huchra, J. P. 1982, ApJ, 257, 23

Beers, T. C., Flynn, K., \& Gebhardt, K. 1990, AJ, 100, 32

Beers, T. C., Forman, W., Huchra, J. P., Jones, C., \& Gebhardt, K. 1991, AJ, 102, 1581

Belsole, E., Pratt, G. W., Sauvageot, J.-L., \& Bourdin, H. 2004, A\&A, 415, 821 Belsole, E., Sauvageot, J.-L., Pratt, G. W., \& Bourdin, H. 2005, A\&A, 430, 385 Bertin, E., \& Arnouts, S. 1996, A\&AS, 117, 393

Biviano, A., Durret, F., Gerbal, D., et al. 1996, A\&A, 311, 95

Boschin, W., Girardi, M., Barrena, R., et al. 2004, A\&A, 416, 839
Bourdin, H., \& Mazzotta, P. 2008, A\&A, 479, 307

Bourdin, H., Sauvageot, J.-L., Slezak, E., Bijaoui, A., \& Teyssier, R. 2004, A\&A, 414, 429

Busha, M. T., Evrard, A. E., Adams, F. C., \& Wechsler, R. H. 2005, MNRAS, 363, L11

Coenda, V., Muriel, H., Donzelli, C. J., et al. 2006, AJ, 131, 1989

Cypriano, E. S., Sodré, L. Jr., Kneib, J.-P., \& Campusano, L. E. 2004, ApJ, 613, 95

Donnelly, R. H., Forman, W., Jones, C., et al. 2001, ApJ, 562, 254

Faltenbacher, A., Jing, Y. P., Li, C., et al. 2008, ApJ, 675, 146

Ferrari, C., Maurogordato, S., Cappi, A., \& Benoist, C. 2003, A\&A, 399, 813

Ferrari, C., Benoist, C., Maurogordato, S., Cappi, A., \& Slezak, E. 2005, A\&A, 430,19

Gardini, A., Rasia, E., Mazzotta, P., et al. 2004, MNRAS, 351, 505

Girardi, M., Giuricin, G., Mardirossian, F., Mezzetti, M., \& Boschin, W. 1998, ApJ, 505, 74

Hartigan, J. A., \& Hartigan, P. M. 1985, Ann. Stat. 13, 1, 70

Jammal, G., \& Bijaoui, A. 2004, Signal Processing, 84, 1049

Katgert, P., Mazure, A., Perea, J., et al. 1996, A\&A, 310, 8

Kolokotronis, V., Basilakos, S., Plionis, M., \& Georgantopoulos, I. 2001, MNRAS, 320, 49

Krauss, L. M., \& Starkman, G. D. 2000, ApJ, 531, 22

Lee, J., \& Evrard, A. E. 2007, ApJ, 657, 30

López-Cruz, O., Barkhouse, W. A., \& Yee, H. K. C. 2004, ApJ, 614, 679

Lubin, L. M., \& Bahcall, N. 1993, ApJ, 415, L17

Maddox, S. J., Efstathiou, G., Sutherland, W. J., \& Loveday, J. 1990, MNRAS, 243, 692

Markevitch, M., Ponman, T. J., Nulsen, P. E. J., et al. 2000, ApJ, 541, 542

Markevitch, M., Gonzalez, A. H., David, L., et al. 2002, ApJ, 567, L27

Maurogordato, S., Proust, D., Beers, T. C., et al. 2000, A\&A, 355, 848

Maurogordato, S., Ferrari, C., Benoist, C., et al. 2006, in Proc. XLIst Rencontres de Moriond, From dark halos to Light, ed. S. Maurogordato, J. Tran Thanh Van, \& L. Tresse (The Gioi Publishers)

Maurogordato, S., Cappi, A., Ferrari, C., et al. 2008, A\&A, 481, 593

McLachlan, G. J., \& Krishnan, T. 1997, The EM Algorithm and Extensions (Wiley)

McLachlan, G. J., Peel, D., Basford, K. E., \& Adams, P. 1999, J. Statistic Software, 4, 2

Miller, N. A., Owen, F. N., Hill, J. M., et al. 2004, ApJ, 613, 841

Mohr, J. J., Geller, M. J., \& Wegner, G. 1996, AJ, 112, 1816

Muriel, H., Quintana, H., Infante, L., Lambas, D. G., \& Way, M. J. 2002, AJ, 124,1934

Nagamine, K., \& Loeb, A. 2003, New Astron., 8, 439

Navarro, J. F., Frenk, C. S., \& White, S. D. M. 1996, ApJ, 462, 563

Niederste-Ostholdt, M., Strauss, M., Dong, F., Koester, B. P., \& McKay, T. A. 2010, MNRAS, 405, 2023

Owers, M. S., Nulsen, P. E. J., Couch, W. J., Markevitch, M., \& Poole, G. B. 2009, ApJ, 692, 702

Pinkney, J., Roettiger, K., Burns, J. O., \& Bird, C. M. 1996, ApJS, 104, 1

Plionis, M., Benoist, C., Maurogordato, S., Ferrari, C., \& Basilakos, S. 2003, ApJ, 594, 144

Poole, G. B., Fardal, M. A., Babul, A., et al. 2006, MNRAS, 373, 881

Popesso, P., Bohringer, H., Romaniello, M., et al. 2005, A\&A, 433, 415

Richstone, D. O., Loeb, A., \& Turner, E. L. 1992, ApJ, 393, 477

Ricker, P. M., \& Sarazin, C. L. 2001, ApJ, 561, 621

Roettiger, K., Loken, C., \& Burns, J. O. 1997, ApJ, 109, 307

Sauvageot, J. L., Belsole, E., \& Pratt, G. W. 2005, A\&A, 444, 673

Schindler, S., \& Böhringer, H. 1993, A\&A, 269, 83

Schlegel, D. J., Finkbeiner, D. P., \& Davis, M. 1998, ApJ, 500, 525

Suto, Y., Sasaki, S., \& Makino, N. 1998, ApJ, 509, 544

Tonry, J. L., \& Davis, M. 1981, ApJ, 246, 666

Teyssier, R. 2002, A\&A, 385, 337

Ulmer, M. P., \& Cruddace, R. G. 1982, ApJ, 258, 434

Vandame, B. 2002, SPIE Proc., 4847, 123

Vikhlinin, A., Markevitch, M., \& Murray, S. S. 2000, ApJ, 551, 160

Way, M. J., Quintana, H., Infante, L., Lambas, D. G., \& Muriel, H. 2005, AJ, 130,2012

West, M. J., Jones, C., \& Forman, W. 1995, ApJ, 451, L5

Wu, X.-P., Fang, L.-Z., \& Xu, W. 1998, A\&A, 338, 813 